\title{
The Recognition Heuristic in Memory-Based Inference: Is Recognition a Non-Compensatory Cue? ${ }^{\dagger}$
}

\author{
THORSTEN PACHUR ${ }^{*}$, ARNDT BRÖDER ${ }^{2}$ and JULIAN N. MAREWSKI ${ }^{3}$ \\ ${ }^{1}$ Cognitive and Decision Sciences, Faculty of Psychology, University of Basel, Basel, \\ Switzerland \\ ${ }^{2}$ Department of Psychology, University of Bonn, and Max Planck Institute for \\ Research on Collective Goods, Bonn, Germany \\ ${ }^{3}$ Center for Adaptive Behavior and Cognition, Max Planck Institute for Human \\ Development, Berlin, Germany
}

\begin{abstract}
The recognition heuristic makes the strong claim that probabilistic inferences in which a recognized object is compared to an unrecognized one are made solely on the basis of whether the objects are recognized or not, ignoring all other available cues. This claim has been seriously challenged by a number of studies that have shown a clear effect of additional cue knowledge. In most of these studies, either recognition knowledge was acquired during the experiment, and/or additional cues were provided to participants. However, the recognition heuristic is more likely to be a tool for exploiting natural (rather than induced) recognition when inferences have to be made from memory. In our study on natural recognition and inferences from memory, around $85 \%$ of the inferences followed recognition information even when participants had learned three cues that contradicted recognition and when some of the contradictory cues were deemed more valid than recognition. Nevertheless, there were strong individual differences in the use of recognition. Whereas about half of the participants chose the recognized object regardless of the number of conflicting cues - suggestive of the hypothesized noncompensatory processing of recognition - the remaining participants were influenced by the additional knowledge. The former group of participants also tended to give higher estimates of recognition's validity. In addition, we found that the use of recognition for an inference may be affected by whether additional cue knowledge has been learned outside or within the experimental setting. Copyright (C) 2007 John Wiley \& Sons, Ltd.
\end{abstract}

KEY WORDS recognition heuristic; decision-making; noncompensatory strategies; memory

\footnotetext{
* Correspondence to: Thorsten Pachur, Cognitive and Decision Sciences, University of Basel, Missionsstrasse 60/62, 4055 Basel, Switzerland. E-mail: Thorsten.Pachur@unibas.ch

${ }^{\dagger}$ This article presents collaborative work of proponents (T.P., J.N.M.) and a skeptic (A.B.) of the recognition heuristic. Although minor disagreements remain on aspects of interpretation and emphasis, the text represents compromise statements that are acceptable to all authors.
}

Copyright (C) 2007 John Wiley \& Sons, Ltd. 


\section{INTRODUCTION}

One important insight into how boundedly rational agents simplify a decision task is the notion that they use noncompensatory strategies (Einhorn, 1970; Keeney \& Raiffa, 1993; Simon, 1955; Tversky, 1972). Rather than integrating all the available cue information-and thus allowing a low value on one cue to be compensated for by a high value on another-noncompensatory strategies ignore some of the information. From a review of 45 process-tracing studies, Ford, Schmitt, Schechtman, Hults, and Doherty (1989) concluded that noncompensatory strategies actually represent the dominant mode used by decision makers. ${ }^{1}$

Goldstein and Gigerenzer (2002) proposed a particularly simple noncompensatory strategy: the recognition heuristic. This fast and frugal decision rule is assumed to be part of an 'adaptive toolbox', from which decision strategies are selected according to the current task environment (Gigerenzer, Todd, \& the ABC Research Group, 1999). The recognition heuristic was proposed for the task of inferring-based on probabilistic cues (i.e. known features of the objects that are correlated with the objects' criterion values) which of two objects has a higher value on a quantitative criterion. The heuristic is an elaboration of an idea developed earlier by Gigerenzer and Goldstein (1996); they proposed that when recognition is a valid cue in the reference class and when cues are not provided by the experimenter (i.e. which would be an inference from givens) but have to be retrieved from memory (i.e. inference from memory), inferences are often based exclusively on recognition. ${ }^{2}$ To illustrate: suppose you are asked which of two German cities has a higher population, Frankfurt or Koblenz. If you have never heard of Koblenz but are familiar with the city name Frankfurt (and do not know its population size), the recognition heuristic predicts that you will judge Frankfurt to have a larger population. This choice would be rather smart because recognition is often correlated with quantity in the environment, in this case, city size. Indeed recognition has been shown to be a valid predictor in many domains, such as geographical quantities (Goldstein \& Gigerenzer, 2002; Pohl, 2006), quality of American colleges (Hertwig \& Todd, 2003), success in sports (Pachur \& Biele, 2007; Scheibehenne \& Bröder, 2007; Serwe \& Frings, 2006; Snook \& Cullen, 2006), political elections (Marewski, Gaissmaier, Dieckmann, Schooler, \& Gigerenzer, 2005) and to some extent, disease incidence rates (Pachur \& Hertwig, 2006).

The important point is that Goldstein and Gigerenzer (2002) proposed the recognition heuristic as a noncompensatory strategy: 'No other information about the unrecognized object is searched for and, therefore, no other information can reverse the choice determined by recognition' (p. 82). In other words, even when people have access to additional relevant cue knowledge-by which we mean knowledge about an object's value on a probabilistic cue that indicates its criterion value-search is stopped after assessing recognition of the two objects, and the recognized object is chosen. For instance, in the German cities example, other characteristics of the cities that are correlated with their size (e.g. whether they have an international airport) are ignored. The recognition heuristic thus predicts that the recognized object is chosen even when other cue knowledge is known that suggests that the recognized object has a small criterion value.

There a number of reasons why in probabilistic inference recognition might be used in a noncompensatory fashion. First, there is evidence that compared to further cue knowledge recognition has a retrieval primacy (Pachur \& Hertwig, 2006). Specifically, whereas recognition is generated by the cognitive system relatively automatically, further cues have to be explicitly recollected, incurring higher cognitive effort. Second, people might refrain from looking up further cues because in situations in which the recognition heuristic is applicable, the cue value of the unrecognized option is usually not known. As a consequence, additional cue

\footnotetext{
${ }^{1}$ Note, however, that conclusions based on process-tracing studies have been criticized (Bröder, 2000a).

${ }^{2}$ We use the term 'recognition' here to refer to what can be called semantic recognition, in contrast to episodic recognition, which is often studied in the recognition memory literature. Episodic recognition refers to the ability to discriminate known objects (i.e. objects one has encountered outside the experimental context, usually words such as HOUSE or GARDEN) that had been previously presented in the experimental context, from other known words that had not been previously presented in the experimental context; semantic recognition refers to the ability to discriminate known objects from (subjectively) novel objects.
} 
knowledge about the recognized object may often be difficult to evaluate. Finally, using computer simulations, Gigerenzer and Goldstein (1996; see their Figure 6) showed that under certain circumstances ignoring further cue knowledge leads to more accurate inferences than considering all available cues.

The thesis that recognition gives rise to noncompensatory processing when it is a valid cue was tested empirically (and, as the authors claimed, corroborated) in Goldstein and Gigerenzer's (2002) second study, but it has been challenged by a number of other studies since then. In this paper, we first briefly review Goldstein and Gigerenzer's (2002) empirical evidence and then offer an overview of other findings conflicting with the claim that recognition gives rise to noncompensatory processing. Second, we discuss possible objections to the conclusions drawn from these challenging results. Third, we present three experimental studies in which we address two major objections: (a) that experimentally induced (rather than natural) recognition might affect the way it is used as a probabilistic cue, and (b) that teaching participants cue knowledge during an experiment might (artificially) enhance its use. ${ }^{3}$ Moreover, in contrast to most previous studies on the recognition heuristic (e.g. Bröder \& Eichler, 2006; Richter \& Späth, 2006; but see Newell \& Shanks, 2004), we not only examine participants' choice behaviour on the aggregate level but also look at possible individual differences in how recognition information is used.

\section{PREVIOUS TESTS OF THE NONCOMPENSATORY STATUS OF RECOGNITION}

In Goldstein and Gigerenzer (2002; Study 2), U.S. participants were informed that in about $78 \%$ of cases German cities that have a soccer team in the premier league are larger than cities that do not. In addition, participants learned whether certain recognized cities had a soccer team or not (nothing was learned about unrecognized cities). In a subsequent inference task in which they were asked to pick the larger of two cities, participants chose a recognized city over an unrecognized one in $92 \%$ of all cases even when they had learned that the recognized city had no soccer team (and thus recognition information was contradicted by additional cue knowledge). According to Goldstein and Gigerenzer, this 'supports the hypothesis that the recognition heuristic was applied in a noncompensatory fashion' (p. 83). This conclusion has been questioned for a number of methodological reasons (summarized in Bröder \& Eichler, 2006; Newell \& Fernandez, 2006 and Richter \& Späth, 2006). The main counter-argument is that one additional cue that is less valid than recognition would not suffice to reverse a recognition-based judgment. Even if people integrate recognition with this cue, their judgments could be indistinguishable from judgments based exclusively on recognition.

A first set of empirical studies challenging the claim that recognition is used in a noncompensatory fashion was presented by Oppenheimer (2003). In Experiment 1, Stanford University students were presented with pairs consisting of small nearby cities, which were highly recognized (e.g. Sausalito), and fictitious cities (a diverse set of made-up foreign-sounding names, such as Heinjing) about which no further knowledge was available. ${ }^{4}$ The task was to decide which city is larger, and here participants' choices often did not conform to the recognition heuristic. In Experiment 2, participants compared the artificial stimuli to cities that were well-known for reasons other than their size (e.g. Chernobyl). Again, the recognized cities were not chosen very often. In both studies participants clearly based their judgments not just on the recognition cue, but also considered additional knowledge (although this knowledge was probably not cue knowledge, but criterion and source knowledge; we will turn to this issue shortly).

Newell and Shanks (2004) attempted to achieve more experimental control over the cue and recognition knowledge that people could use for a probabilistic inference. In two experiments they set up a hypothetical

\footnotetext{
${ }^{3}$ We are aware that the term 'natural' used here repeatedly is a rather fuzzy one since there are no clear defining boundaries between natural and artificial environments (such as the laboratory). Here we use the term for a task which (a) uses real world stimuli and (b) for which the crucial recognition knowledge must have been acquired before the experiment and outside the laboratory.

${ }^{4}$ Note that the names of the fictitious cities were chosen such that they allowed for an informed guess about their country of origin.
} 
stock market in which some artificial company names were presented repeatedly - participants thus learned to recognize these names during the experiment. Participants also learned that this induced recognition was a valid cue to predict the success of stocks. To judge which of two stocks is more profitable, participants were able to acquire - at some cost - advice from three experts, so the experiments involved inferences from givens (rather than inferences from memory). As it turned out, participants often purchased additional information ( $48 \%$ of the time), even when they had seen one company name before but not the other. Moreover, when the purchased advice was favourable to the unrecognized stock, the unrecognized stock was chosen almost $60 \%$ of the time, so this additional information was clearly incorporated into the decision.

Using a methodology similar to that of Newell and Shanks (2004), Bröder and Eichler (2006) repeatedly presented unknown city names to participants. In addition, participants had to memorize further cue knowledge about the cities they learned to recognize. Subsequently they had to decide which of two cities was larger, which, in contrast to Newell and Shanks' experiments, thus represented inferences from memory. The judgments about pairs in which one city was recognized but the other was not showed that the additional cue knowledge had a large impact on the proportion of judgments that followed recognition (see Figure 1a). This finding is inconsistent with the claim that recognition is used in a noncompensatory fashion.

A series of experiments that involved both natural recognition and a memory-based design was reported by Richter and Späth (2006). Participants had to judge the population sizes of animal species (Experiment 1), airline safety (Experiment 2) and city sizes (Experiment 3). Well-recognized and unrecognized names had been identified in pre-studies. Moreover, in all experiments additional knowledge was available (for some recognized objects), and in Experiments 2 and 3 participants acquired cue knowledge in a learning phase. All three experiments showed that the knowledge that participants had in addition to name recognition exerted an influence on their judgments. Figure $1 \mathrm{~b}$ shows the impact of additional cue knowledge in Experiment 2. As in Bröder and Eichler (2006), the proportion of choices of the recognized object increased monotonically with the number of positive cues; again this challenges the claim that recognition is used in a noncompensatory way.

Newell and Fernandez (2006; Experiment 1) replicated Goldstein and Gigerenzer's Study 2 but added a second condition in which the soccer team cue was a more useful indicator of a city's size (specifically, the probability that an unrecognized city had a soccer team was increased). In this second condition, only $55.5 \%$
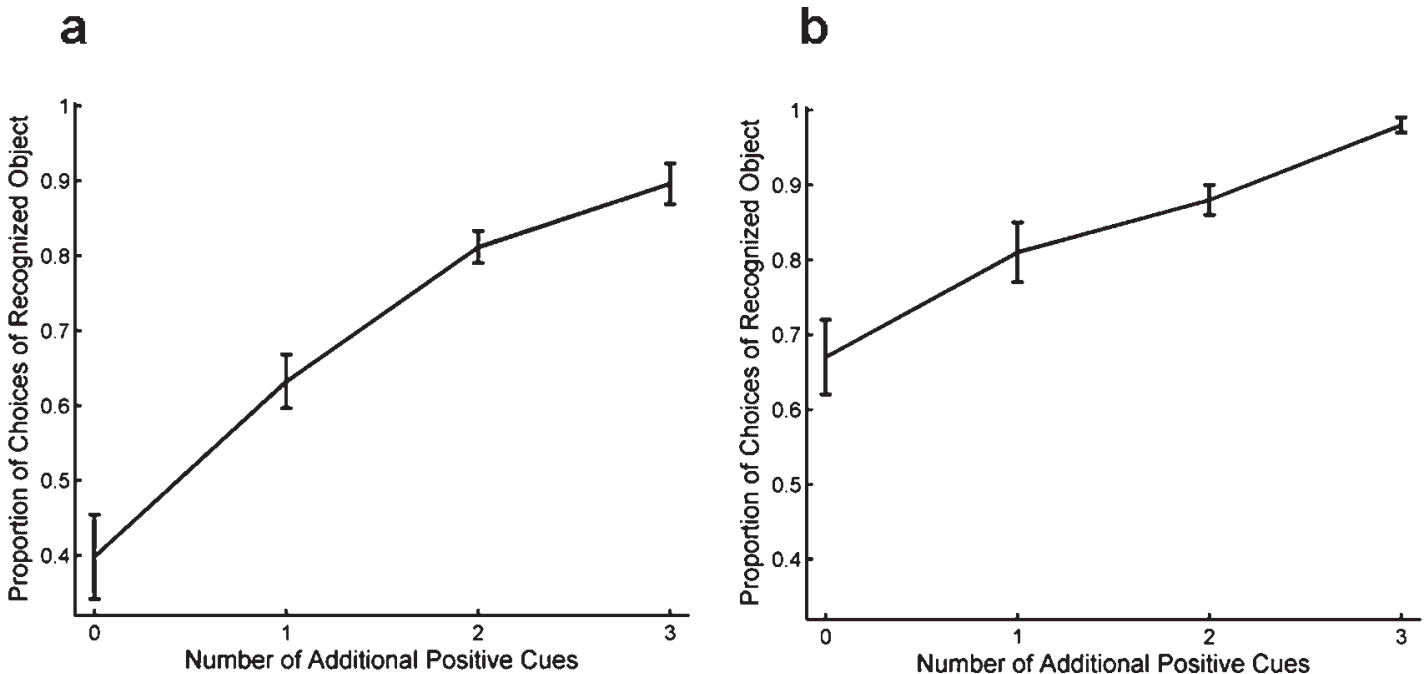

Figure 1. Proportion of choices of recognized object in (a) Bröder and Eichler (2006) and (b) Richter and Späth (2006; Experiment 2). Error bars show \pm 1 standard error 
of the judgments were consistent with recognition, whereas this was the case for $72.6 \%$ of the judgments in the replication of the original study. Hence, the additional cue clearly influenced the judgments.

Last but not least, Pohl (2006) presented four experiments in which Swiss, German, Italian and Belgian cities, as well as mountains, rivers and islands, had to be judged on various quantitative target variables. Based on the objects' recognition ratings and their values on the target variables, Pohl divided the items in the judgment task into two sets: In one set the recognition heuristic led to a correct decision and in the other set to an incorrect decision. Comparing these two sets, Pohl found significant differences in the proportion of judgments that followed recognition. This implies that additional knowledge must have been used, because otherwise, ceteris paribus, the proportions of judgments following recognition would not differ between the two sets of items.

\section{HOW CAN THE RECOGNITION HEURISTIC BE TESTED?}

Note that none of the experiments reviewed denies that recognition (be it binary or continuous; see Newell \& Fernandez, 2006; but see Schooler \& Hertwig, 2005) plays a major role in decision-making. For example, Bröder and Eichler (2006) demonstrated that the recognition cue entailed a large additional effect (partial $\eta^{2}=.52$ ). This effect was obtained when trials where recognized cities for which people had additional cue knowledge were paired with unrecognized cities were contrasted with trials where these recognized cities were paired with merely recognized ones (for which no further cue knowledge was available). However, all experiments (except Goldstein \& Gigerenzer, 2002) claim to show that recognition is not used exclusively and thus that the recognition heuristic is not descriptive of how people make judgments. At the same time, most of these studies tested the recognition heuristic in experimental situations that differed in potentially critical ways from the situations for which Goldstein and Gigerenzer formulated the heuristic. An overview of the methodological differences between previous tests of the noncompensatory use of recognition is shown in Table 1. In the following, we focus on four central issues: the availability of conclusive criterion knowledge, the use of induced recognition, the use of induced cue knowledge and low recognition validity.

\section{Conclusive criterion knowledge}

Goldstein and Gigerenzer (2002) formulated the recognition heuristic for 'cases of inference, [where] the criterion is not immediately accessible to the organism' (p. 78) and where an inference has to be made based on probabilistic cues. In Oppenheimer (2003; Experiment 1), however, it is likely that participants knew the sizes of the recognized nearby cities and that the sizes were very small (e.g. Sausalito has around 7000 inhabitants). If one makes the — not completely implausible - assumption that participants believed that the typical city size in the countries alluded to by the fictitious names (e.g. China) was rather large, this knowledge could be used to deduce that the recognized city is the smaller one. From the perspective of Gigerenzer, Hoffrage, and Kleinbölting's (1991) theory of probabilistic mental models (PMM) one could thus argue that participants might have solved the task based on a local mental model (LMM). In contrast to a PMM, an LMM does not exploit the probability structure of the environment (e.g. the recognition cue; see Gigerenzer et al., 1991, for further details). In other words, in a situation where an inference can be deduced the use of a tool for probabilistic inductive inference is unnecessary (although the decision might still involve some uncertainty). Similarly, in Richter and Späth's (2006) Experiment 1, additional knowledge was defined as knowing whether a species is endangered, which is equivalent to knowing whether a population is very small. Both of these experiments thus might have involved conclusive criterion knowledge (rather than cue knowledge; Pachur \& Hertwig, 2006), defined as direct knowledge of an object's criterion value that locates the object on an extreme (either very high or very low) position on the criterion dimension; conclusive criterion knowledge allows a decision maker to deduce a judgment without engaging in processes of 
Table 1. Methodological differences in previous experiments testing the noncompensatory use of recognition

\begin{tabular}{|c|c|c|c|c|c|c|c|c|c|c|}
\hline & 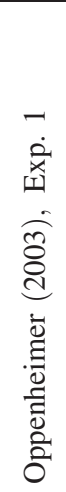 & 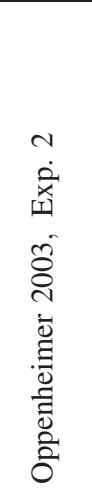 & 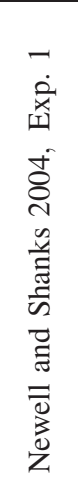 & 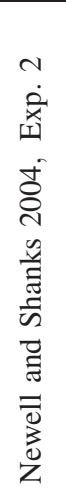 & 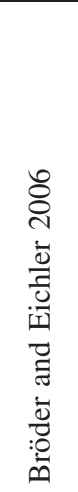 & 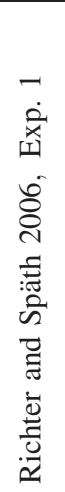 & 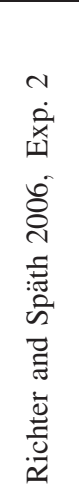 & 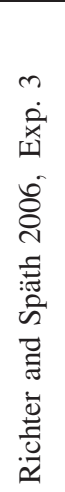 & 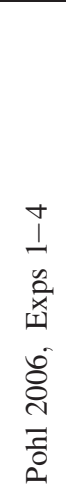 & 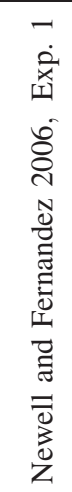 \\
\hline $\begin{array}{l}\text { Induced (rather than natural) recognition } \\
\text { Induced (rather than natural) cue knowledge } \\
\text { Criterion (instead of cue) knowledge } \\
\text { Menu-based (rather than memory-based) inference } \\
\text { Domain with low recognition validity } \\
\text { Unknown nature of additional cue knowledge } \\
\text { Artificial stimuli } \\
\text { Cue knowledge available about unrecognized object }\end{array}$ & X & $(\mathrm{X})^{* *}$ & $\begin{array}{c}\mathrm{X} \\
\mathrm{X} \\
\mathrm{X} \\
(\mathrm{X})^{*}\end{array}$ & $\begin{array}{l}\mathrm{X} \\
\mathrm{X} \\
\mathrm{X}\end{array}$ & $\begin{array}{l}X \\
X\end{array}$ & $\begin{array}{l}X \\
X\end{array}$ & X & $X$ & $X$ & X \\
\hline
\end{tabular}

Notes:

*Newell and Shanks (2004) manipulated the recognition validity experimentally and used both a high validity and a low validity condition.

** Oppenheimer (2003) used a mixture of artificial and real stimuli.

${ }^{\dagger}$ The stimuli used by Bröder and Eichler (2006) were real cities, but were selected for being unrecognized by most participants.

${ }_{\ddagger}^{\ddagger}$ Newell and Fernandez (2006) provided information about the unrecognized object only indirectly, as they manipulated the probability that the unrecognized object has a particular cue value.

inductive inference. For instance, if one knows that a disease is practically extinct, one can deduce that it is less frequent than any other disease. Conclusive criterion knowledge could also-at least partially - have driven the results reported by Pohl (2006), where the nature of the knowledge that was available in addition to recognition information was unclear: Participants merely indicated whether they also had further knowledge about an object, but not whether it was cue or criterion knowledge.

\section{Use of induced recognition}

In both Newell and Shanks (2004) and Bröder and Eichler (2006), recognition was induced during the experiment rather than having been established outside the experimental setting, and participants were able to clearly attribute their recognition knowledge to this manipulation (in Newell and Shanks' experiments participants were even explicitly reminded that their recognition was restricted to the experimental context; see p. 926). As the ecological rationality of the recognition heuristic lies in its 'ability to exploit the structure of the information in natural environments' (Goldstein \& Gigerenzer, 2002, p. 76, emphasis added), experimenter-induced recognition may not be the appropriate test-bed for the heuristic (although it is of course possible that a heuristic evolved in natural environments is also applied in artificial ones). As is well-known for other assessments of memory (such as fluency or the number of recalled instances), people use such assessments considerably less to infer quantities outside the laboratory situation when they believe that memory has been manipulated experimentally (e.g. Jacoby, Kelley, Brown, \& Jasechko, 1989; 
Oppenheimer, 2004; Schwarz, Bless, Strack, Klumpp, Rittenauer-Schatka, \& Simons, 1991)—or, more generally, when they can pin down the source of the memory to one specific factor. It is thus conceivable that, analogously, people rely less on recognition that was induced in the laboratory than on recognition that evolved outside the experimental setting (where one's recognition typically cannot be attributed exclusively to a specific source). As a consequence, in tests of the recognition heuristic using induced (rather than natural) recognition, recognition information could be underweighted. Consistent with this hypothesis, Marewski, Gaissmaier, Schooler, Goldstein, and Gigerenzer (2007) observed that experimentally induced recognition was followed less in inferential judgments when participants knew that it was induced compared to when they falsely believed that they had acquired it outside the laboratory.

Note, however, that the fact that natural and induced forms of recognition seem to be treated differently for inferences implies that people do indeed take the source of recognition (if a specific one sticks out) into account - a process that Goldstein and Gigerenzer did not consider in their original model. Such attribution or evaluation processes are also apparent in Oppenheimer (2003; Experiment 2), where people only rarely relied on recognition of cities they knew because of a factor that was clearly unrelated to the city's size. Evidence for the involvement of evaluation processes was also provided by a functional magnetic resonance imaging (fMRI) study by Volz, Schooler, Schubotz, Raab, Gigerenzer, \& Cramon (2006), who observed neural activity that is usually associated with metacognitive evaluation processes in situations in which the recognition heuristic could be applied.

\section{Use of induced cue knowledge}

All the above reviewed experiments in which additional knowledge was controlled involved induced cue knowledge. That is, participants were instructed to memorize cue knowledge immediately before making their inferences (Newell \& Fernandez, 2006; Bröder \& Eichler, 2006; Goldstein \& Gigerenzer, 2002; Richter \& Späth, 2006, Experiments 2 and 3) or the cues were provided to them on a computer screen (Newell \& Shanks, 2004). To be sure, this procedure allowed the experimenter to control which information participants could use for an inference. Nevertheless, inducing cue knowledge can potentially increase participants' tendency to search and use additional cue knowledge due a demand effect. Specifically, the participant might ask: 'Why would the experimenter provide a cue if I am not supposed to use it?' (e.g. Grice, 1975; Hilton, 1995); teaching the cue values might thus make participants pay more attention to additional cue knowledge than they usually would. As regards the menu-based procedure used by Newell and Shanks (2004), where cue knowledge does not have to be retrieved from memory but is presented on the computer screen, it has been shown that the use of noncompensatory decision strategies such as Take The Best (TTB) is much more prevalent in memory-based than in menu-based decisions (Bröder \& Schiffer, 2003, 2006). Taken together, inducing cue knowledge in the experiment might lead to an overweighting of this knowledge.

\section{Low recognition validity}

According to the notion of an adaptive toolbox, whether the recognition heuristic is selected depends on the current task environment. One central aspect of the task environment is the validity of the recognition cue in the reference class from which the items are drawn (see Gigerenzer et al., 1991). Accordingly, one should assume that the heuristic is used in particular when recognition is a good predictor of the criterion, but considerably less when recognition is only slightly better than chance. (In fact, recognition might be used in more complex ways when its validity is low. For a test of possible mechanisms for suspending the use of recognition, see Pachur \& Hertwig, 2006.) Pohl (2006) provides some evidence in support of this assumption. One can thus argue that in Richter and Späth's (2006) Experiments 1 and 2, the recognition heuristic (i.e., using recognition as the sole basis for a judgment) was not an adaptive strategy. In Experiment 1, where participants judged the population size of animal species, the recognition validity was probably low since 
both very common and endangered (and thus very rare) species are well-known. In Experiment 2, the correlation between recognition rates and airline casualties per person miles was -.28 only after controlling for the year in which the airline was established. First, this correlation is rather low, and second, controlling for the year in which the airline was established seems unwarranted, since this information is usually unavailable to participants.

In our view, those studies that might have involved conclusive criterion knowledge (rather than cue knowledge) and those that used a low-validity domain are the least critical tests of the recognition heuristic (Oppenheimer, 2003, Experiment 1; Richter \& Späth, 2006, Experiments 1 and 2). When conclusive criterion knowledge is available, no probabilistic inference is required. In domains with low recognition validity, the use of the heuristic is not adaptive-although we agree that studying people's use of the recognition heuristic in such environments is important for testing the notion of adaptive decision-making. Two further studies involved experimentally induced rather than naturally acquired recognition (Bröder \& Eichler, 2006; Newell \& Shanks, 2004). Finally, all studies that pitted recognition against contradictory cue knowledge involved induced rather than natural cue knowledge (Bröder \& Eichler, 2006; Goldstein \& Gigerenzer, 2002; Newell \& Fernandez, 2006; Newell \& Shanks, 2004; Richter \& Späth, 2006, Experiments 2 and 3). We submit that teaching cue knowledge to participants in the context of the experimental setting might create a demand effect and thus artificially enhance the use of additional cues. Of the previous experiments, Richter and Späth's (2006) Experiment 3 represents, in our view, the best test hitherto conducted of the claim that recognition is used in a noncompensatory way. This experiment involved the task of judging city sizes, a domain where a high recognition validity has been established (see Goldstein \& Gigerenzer, 2002), along with natural recognition and a memory-based procedure. The only critical point concerns the teaching of cue knowledge, which could create a demand effect. We will address this objection in our Experiment 3.

Before we go on, two comments are in order. First, even if we argue that some of the criticism of the recognition heuristic may be unwarranted, we do not wish to devalue the studies that fuelled the criticism. Rather, we see them as important tests of how far the idea of noncompensatory processing of recognition can be taken; these tests identified a number of important moderating factors, some of which had been anticipated by Goldstein and Gigerenzer (2002). Second, in spite of our reservations concerning some of the evidence against the recognition heuristic, we still think it is necessary to revise the original model. We see increasing evidence that people do evaluate the validity of recognition for a specific decision situation (e.g. Oppenheimer, 2003, Experiment 2; Volz et al., 2006). Goldstein and Gigerenzer's original version of the model, however, is mute in this regard, or assumes that the source of one's recognition is disregarded (cf. Goldstein \& Gigerenzer, 2002, p. 76). For a more veridical description of how recognition is used for inferences, a revision of the recognition heuristic should make these processes more explicit. In this paper, however, we focus on testing the heuristic under the circumstances that fit the purposes of the heuristic as it was originally formulated. Under these circumstances, do people follow recognition even when substantive evidence points against it?

\section{OVERVIEW OF THE EXPERIMENTS}

Our general approach to testing the noncompensatory use of recognition was as follows: We contrasted the situation in which an unrecognized city was compared with a recognized city and the participants had cue knowledge indicating that the recognized city was small with the situation in which participants had cue knowledge indicating that the recognized city was large. To avoid the potential limitations of earlier studies outlined in the previous section, in our experiments we used naturally acquired recognition about real objects in a high-validity domain (cities) and assessed the impact of cue knowledge in a memory-based setting. Experiments 1 and 2 assessed participants' use of additional cue knowledge taught in the laboratory, whereas Experiment 3 assessed the impact of natural (rather than induced) cue knowledge, which participants had 
acquired outside the experimental situation. The relevant cues were determined in a pre-study with a different sample of participants. In our view, this constitutes a fair and strict test of the hypothesis that recognition is used in a noncompensatory fashion. We thus strove (a) to examine whether additional cue knowledge affects the proportion of cases where a recognized city is chosen over an unrecognized one in a situation in which recognition is natural (while, in Experiments 1-2 manipulating the additional cue knowledge); and (b) to assess the effect of inducing cue knowledge by comparing results to a situation where cue knowledge is not manipulated. Although most previous studies only looked at choices on an aggregate level, we also analysed participants individually. An analysis on the individual level may be important as even when only a minority of participants use recognition in a compensatory way (whereas all others use it in a noncompensatory fashion), on the aggregate level it may look as if recognition is generally used in a compensatory way.

In Experiment 1, participants learned additional cue knowledge about cities that - as shown in a pre-study with German participants - were highly recognized and for which relatively little cue knowledge was available. In Experiment 2, the amount of cue knowledge contradicting recognition was increased. Importantly, at no point during the learning or the judgment phases of Experiments 1 and 2 was the potential usefulness of the cues for making inferences about the cities mentioned; rather, we relied on participants' subjective assessment of the relevance of these cues. Participants' subsequent estimates of the cue validities confirmed that the selected cues were indeed perceived to be valid indicators of the size of British cities. Nevertheless, teaching cue knowledge might still artificially enhance the use of additional cue knowledge. Therefore, in Experiment 3, rather than teaching cue knowledge to participants, we examined the impact of pre-existing cue knowledge. In all three experiments, we obtained participants' subjective validity ratings for the cues involved, allowing, to the best of our knowledge for the first time, the comparison of the perceived validities of natural recognition and other cues.

\section{EXPERIMENT 1: DOES INDUCED CUE KNOWLEDGE OVERRIDE NATURAL RECOGNITION?}

The first experiment had the goal of replicating the results found by Bröder and Eichler (2006) using natural rather than induced recognition. We thus addressed the possibility that induced recognition is treated differently from natural recognition (i.e. recognition knowledge that arises through the natural process of encountering objects in the real world). We predicted that if participants trust their natural recognition knowledge more than experimentally induced recognition, additional cue knowledge should be less likely to overturn recognition in people's inferences.

\section{Method}

\section{Participants}

Forty students (19 females; mean age $=24.6$ years) participated in the experiment, which was conducted at the Max Planck Institute for Human Development in Berlin. They received a flat fee of $€ 9$ (\$11.50 U.S.) and also an extra payment depending on their performance in the inference and memory tasks (see below).

\section{Materials}

Two pre-studies were conducted to create appropriate materials. In the first pre-study ( $N=100$ participants), we assessed the recognition rates of the 50 largest cities of each of four European countries (France, United Kingdom, Italy, Spain; 25 participants for each country). In addition, to identify cues with a high subjective validity for inferring the population size of European cities, participants were asked to provide, in a free-answer format, cues they would consider ('Which characteristics might be useful to distinguish between large and small cities?'). Based on participants' recognition ratings, we calculated the recognition validity for 
the four reference classes. In line with previous results, the city domain turned out to have a high recognition validity (.78, .74, .76 and .72 for France, United Kingdom, Italy and Spain, respectively). Because the recognition rates varied most markedly among the British and Italian cities, we retained these two city sets for the second pre-study. The cues mentioned most often as useful for inferring city size were the existence of an international airport (mentioned by $43 \%$ of the participants), the existence of significant industry (42\%), a university (37\%), a world-famous tourist site (34\%) and a team in the major national soccer league (30\%).

In the second pre-study (with $N=60$ participants), we assessed cue knowledge and subjective validity ratings for the cities and 11 cues identified in the first pre-study respectively. The 15 most and 15 least recognized cities (as identified in Pre-study 1) from the United Kingdom and Italy were used. The cities of London and Rome were excluded as they are probably known to be the largest British and Italian cities, respectively (which would represent conclusive criterion knowledge and thus allow participants to solve an inference based on an LMM). Participants indicated whether a city possessed a particular feature (e.g. international airport, responses 'yes', 'no', or 'don't know'). Later, the concept of cue validity was explained and participants estimated the validities of the five cues on a scale ranging from $50 \%$ to $100 \%$. The three cues deemed as most valid turned out to be industry $(M=81.1 \%)$, airport $(M=80.3 \%)$ and soccer team $(M=71.9 \%)$. These cues were chosen for the experiments.

The cities that were selected as critical stimuli for the experiments had to meet two criteria: In order to create many pairs comparing recognized with unrecognized cities, we chose homogenous subsets of cities that were recognized either most of the time $(>75 \%)$ or very rarely $(<15 \%)$. In addition, we aimed for the additional cue knowledge for a given recognized city being homogenous, too. To achieve this, the cue value assigned to a city on a given cue most frequently had to be assigned (to that city) at least 20 percentage points more frequently than the cue value assigned second-most frequently. Since the latter criterion was met by merely four Italian cities, we decided to use the British cities only. Altogether eight highly recognized (i.e. recognized by $>75 \%$ of the participants; ' $R$ ' cities) British cities were chosen, as well as 10 unknown (i.e. recognized by $<15 \%$ of the participants; ' $U$ ' cities; see Table A1 in the Appendix) British cities. These two types of cities were used to create the critical (i.e. RU) city pairs, for which we expected that participants would recognize one, but not the other (in the analysis of the experiments we took participants' actual recognition responses into account). In addition, a separate set of five highly recognized cities was used to create filler items. For two of the $\mathrm{R}$ cities (Manchester and Liverpool), the most frequently assigned cue value was 'yes' for all three cues. For the other six R cities (Aberdeen, Bristol, Brighton, Nottingham, Sheffield, York), the most common value for all cues was 'don't know'. Given that for these six cities pre-existing cue knowledge (which could interfere with learned cue knowledge) was relatively rare, in Experiments 1 and 2 cue values were learned for them. The cue values that the participants learned were those that were indicated most frequently (after 'don't know') in the second pre-study (in Experiment 2 these cue patterns were slightly modified). To increase the likelihood that participants would have knowledge of the relevant cue dimensions, Aberdeen and Bristol were replaced by Manchester and Liverpool in Experiment 3, which examined how natural cue knowledge influences the use of recognition in inference.

Importantly, although not specifically selected for this purpose, the cities of York and Brighton had the desirable property of being rather small. In fact, they were the 46th and 50th largest cities (of the 50 largest cities), and thus smaller than most of the unrecognized cities. It is thus not the case simply that the R cities were large and the U cities were small, which could confound recognition with knowledge of a city's size (but note that this 'confound' is what makes recognition a powerful cue in many domains).

\section{Design and procedure}

The experiment started with a learning task, in which participants acquired cue knowledge about six cities. The task was described as involving the learning of geographical facts about British cities. The cue knowledge consisted of the cue values (either 'yes' or 'no') that were indicated most frequently in Pre-study 2 
Table 2. Cue profiles of the cities used in Experiments 1-3 (the proportion of responses for these cue values in Experiment 3 are given in parentheses)

\begin{tabular}{|c|c|c|c|c|c|c|c|c|}
\hline \multirow[b]{2}{*}{ Cue } & \multicolumn{8}{|c|}{ City } \\
\hline & Aberdeen* & Bristol $^{*}$ & Manchester** & Liverpool $^{* *}$ & Nottingham & Sheffield & Brighton & York \\
\hline Industry & Yes & Yes & Yes (0.97) & Yes (1) & Yes $(0.50)$ & Yes (0.67) & $\mathrm{Yes}^{\dagger}(0.64)$ & $\mathrm{Yes}^{\dagger}(0.69)$ \\
\hline Airport & Yes & Yes & Yes $(0.91)$ & Yes $(0.83)$ & No $(0.80)$ & No $(0.91)$ & No $(0.66)$ & No $(0.80)$ \\
\hline Soccer & Yes & Yes & Yes (0.97) & Yes (0.97) & Yes (0.57) & Yes $(0.56)$ & No (0.69) & No $(0.55)$ \\
\hline $\begin{array}{l}\text { Number of } \\
\text { positive cues }\end{array}$ & 3 & 3 & 3 & 3 & 2 & 2 & 1 & 1 \\
\hline
\end{tabular}

Note: In Experiments 1 and 2, the cues values were learned during the experiment.

*Only used in Experiments 1-2.

** Only used in Experiment 3.

'In Experiment 2, the values of Brighton and York on the industry cue were replaced by 'No'.

(see Table 2). A positive cue value (='yes') suggests that the city is large, and a negative cue value (='no') that the city is small. Note that no city had negative cue values on all three cues. We used an anticipation learning paradigm, which proceeded as follows (cf. Bröder \& Eichler, 2006; Bröder \& Schiffer, 2003). At every learning trial, participants were presented with a city and a cue on a computer screen and instructed to find out the correct cue value by clicking on either 'yes' or 'no', in the beginning often by simply guessing. Immediately after a response, they received feedback and were asked to memorize the correct value (they were informed that they would be tested again later). Then the next cue followed (for the same city; see Figure 2). In contrast to previous studies (e.g. Bröder \& Eichler, 2006; Richter \& Späth, 2006) the sequence of cues was determined at random and changed with every learning cycle. By this procedure, we wanted to reduce the possibility that after learning, the cue profiles would be represented as chunks rather than as individual cue values. After the third cue, the procedure was repeated (with the cues presented in a new sequence) until all cue values for the city had been correctly reproduced twice in a row. Then the next city followed. After the last city, the procedure was repeated (in a new random order) until the participants had reproduced all cue values for all cities without error twice in a row. The potential usefulness of the cues for inferring the size of British cities was mentioned at no point during the learning task.

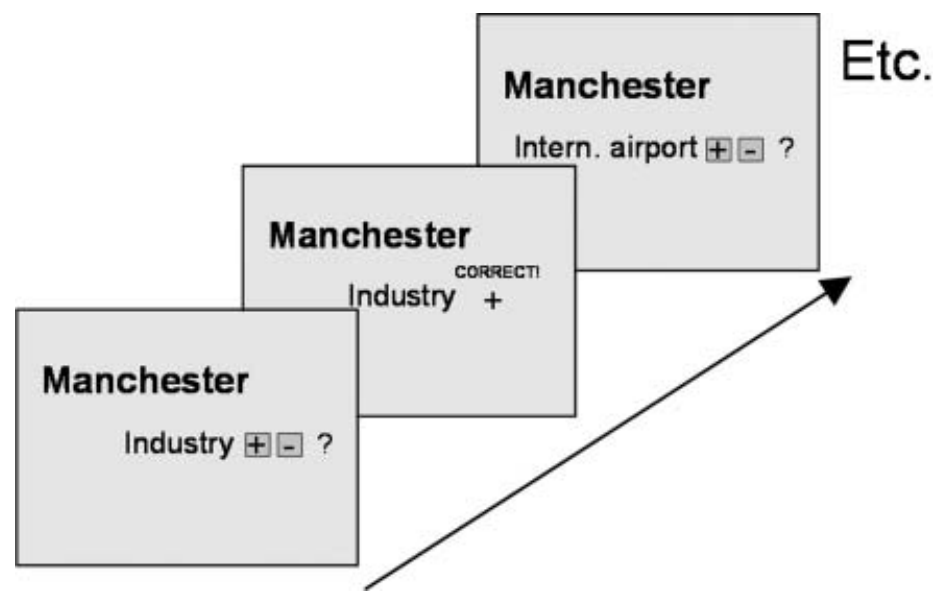

Figure 2. Procedure used in the cue-learning task 
After having learned the cue values perfectly, participants performed an inference task in which they were presented with a total of 120 pairs of British cities (in blocks of 20 pairs, after each of which participants could take a short break) and instructed to choose the one with more inhabitants (no feedback was given). For each trial, a pair was drawn at random from one of three item types. First, the six recognized cities were combined with all 10 unrecognized cities, yielding 60 RU pairs. These 60 pairs were critical for our purposes. Second, there were 30 pairs consisting of two unrecognized cities (UU pairs), drawn at random from all possible pairings of the 10 unrecognized cities. Third, to equalize the presentation frequency of the $\mathrm{R}$ and $\mathrm{U}$ cities as much as possible, there were 30 pairs with two recognized cities, drawn at random from a pool consisting of the six $\mathrm{R}$ cities and the five filler cities. As a result, each of the $\mathrm{R}$ cities was presented, on average, 11.5 times, the $U$ cities 12 times and the filler cities 5.5 times. For each correct inference (according to the official statistics ${ }^{5}$ ), participants earned an additional $4 \notin$ ( $5 \varnothing$ U.S.), and the same amount was subtracted for every incorrect inference.

The inference task was followed by a recognition task. Participants were presented with the 21 cities (6 R, $10 \mathrm{U}$ and 5 filler cities) in a random order and asked to indicate whether they had heard of them before participating in the experiment. After the recognition task, participants performed a memory task in which they had to reproduce the cue values ('yes' or 'no') they had learned for the six R cities in the learning task. If they could not recall the correct values, they were allowed to respond 'don't know'. A 'no' response thus did not simply mean that the participants could not remember the city having a positive cue value. Every correctly recalled cue value earned them $10 \notin(13 \notin$ U.S.), and $10 \notin$ was subtracted for every incorrect answer and 'don't know' response. Finally, in a cue validity estimation task, participants judged the validities of the four cues (the three cues identified in the pre-study and recognition, presented in a random order) for the task of inferring the population of British cities (using a frequentistic format, ranging from $50 \%$ to $100 \%$ ).

\section{Results and discussion}

Cue knowledge, recognition and cue validity estimates

On average, participants took 12.4 minutes $(S D=7.1)$ to learn the 18 ( 3 cues $\times 6$ cities) cue values perfectly. The mean accuracy in the subsequent memory task (after the inference task) was $95 \%(S D=7$; range 72-100\%). Twenty of the 40 participants recalled the cue values perfectly. The option 'don't know' was chosen in $1 \%$ of the responses. Of the 60 critical pairs (i.e. the RU pairs), the mean proportion of comparisons where one city was recognized and the other not was .82 $(S D=0.16)$. To address the possible objection that the repeated presentation of previously unrecognized cities during the learning and inference tasks might distort the responses in the recognition task, in Figure A1 in the Appendix the recognition rates obtained in Experiments 1-3 for the individual cities are compared with the respective rates obtained in Pre-study 1 (where participants saw the cities only once). As can be seen, if there are any differences at all, they are very small, suggesting that participants are very capable of disregarding their experimental familiarity with the city names. Concerning the estimated cue validities, Table 3 shows that all four cues were estimated to be relevant, with the international airport cue deemed to be the most valid one (replicating Richter \& Späth, 2006), followed by the industry site cue, recognition and the soccer team cue. ${ }^{6}$ Importantly, the airport cue was estimated to be significantly more valid than recognition, $t(39)=4.18, p=.001$. We thus had the opportunity to submit the recognition heuristic to a tough test, since according to the cue patterns that the participants learned, the airport cue contradicted recognition. Would participants follow recognition even when it was contradicted by a cue deemed as more valid than recognition?

\footnotetext{
${ }^{5}$ Relevant statistics were obtained from: http://www.citypopulation.de/UK.html (retrieved on 15 April 2005).

${ }^{6} \mathrm{An}$ analysis of the cues' actual, that is, their ecological validities (cf. Gigerenzer et al., 1991) indicated that these estimates were very accurate: for the reference class of the 50 largest British cities the ecological validities for the industry, airport and soccer cues were .78, .85 and .69 , respectively. The average recognition validity in Pre-study 1 was .74 .
} 
Table 3. Estimated validities (expressed as the percentage of correct inferences when the cue value is positive) of recognition and the three cues deemed most valid in the pre-studies

\begin{tabular}{llcccc}
\hline & & Industry & Airport & Soccer & Recognition \\
\hline Experiment 1 & $M$ & 75.0 & 82.1 & 63.5 & 70.2 \\
& $S D$ & 14.7 & 11.4 & 11.1 & 13.4 \\
Experiment 2 & $M$ & 77.7 & 83.3 & 63.4 & 72.2 \\
& $S D$ & 15.5 & 11.1 & 11.7 & 12.6 \\
Experiment 3 & $M$ & 78.5 & 83.3 & 61.5 & 71.3 \\
& $S D$ & 12.8 & 8.4 & 9.9 & 13.6 \\
\hline
\end{tabular}

Effect of additional cue knowledge on choice of recognized city

Overall, participants' judgments followed recognition in, on average, 95.6\% ( $S D=0.08)$ of the critical RU pairs where one city was recognized but the other was not. Did this figure vary as a function of the number of additional positive and negative cue values known? Depending on the responses in the memory task, each item was categorized according to the number of cue values - positive or negative-known about the R city. There were three categories: 1,2 , or 3 cues.

To test for an effect of additional cue knowledge, we collapsed the choices of all participants and compared the proportion of choices of the recognized city across the three different categories. Recall that the recognition heuristic predicts that further cue knowledge does not affect the choice of the recognized object. In line with this prediction, we did not find the proportion of choices of the recognized city to differ across the varying levels of additional cue knowledge, and this was true for both positive $\left[\chi^{2}(2, N=1947)=2.61\right.$, $p=.27$; two-tailed; $w=.04]$ and negative cue knowledge $\left[\chi^{2}(2, N=1958)=0.55, p=.76\right.$; two-tailed; $w=.02]$. Figure $3 \mathrm{a}$ shows the proportion of choices of the recognized city across the different levels of additional cue knowledge (1,2 or 3 positive, and 1, 2 or 3 negative cues, respectively). The results for positive and negative cue values are shown separately because, due to 'don't know' responses, the two could potentially diverge (see Experiment 3). As, however, unknown cue values were very rare in this experiment, the results for positive and negative cues are practically symmetrical. To test for a monotonic trend across the different levels of additional cue knowledge, we conducted a trend test developed by Pfanzagl (1974, p. 193). For this test, $\alpha$ was set at .05 , with a corresponding critical threshold of 1.65 . The observed test value $T$ did not reach this critical threshold for positive $(T=1.62)$ or negative $(T=0.65)$ cue values. In an analysis on the individual level we considered only those participants with at least one case where the recognized city had only one additional positive cue (where the influence of the recognition cue could potentially be equalized; the median number of such cases was 16). Of these participants, $60 \%$ (22 of 37) always chose the recognized city.

In sum, in contrast to Bröder and Eichler (2006) and Richter and Späth (2006), we did not find additional cue knowledge to affect the proportion of choices of a recognized object over an unrecognized one. Although one might be quick to attribute the discrepancy between these previous and present findings to methodological differences (in particular, to the fact that we prevented cue values from being learned as chunks), note that the proportion of choices of the recognized city was generally very high, and thus the lack of impact of additional cue knowledge could be due to a ceiling effect. In addition, the test of a monotonic trend failed to reach the critical threshold only by a little ( $T \mathrm{~s}=1.62 \mathrm{vs.} 1.65$, for positive cue values). Finally, one could object that the additional cue knowledge contradicting recognition was too weak to show an effect. Recall that, due to the cue profiles used, the recognized city always had an additional cue supporting recognition (although in both Bröder and Eichler and Richter and Späth, the proportion of choices of the recognized object differed also between 1 and 3 positive cues; see Figure 1). In other words, it is possible that the amount of cue knowledge indicating that the recognized city might be small was not sufficient to overturn 

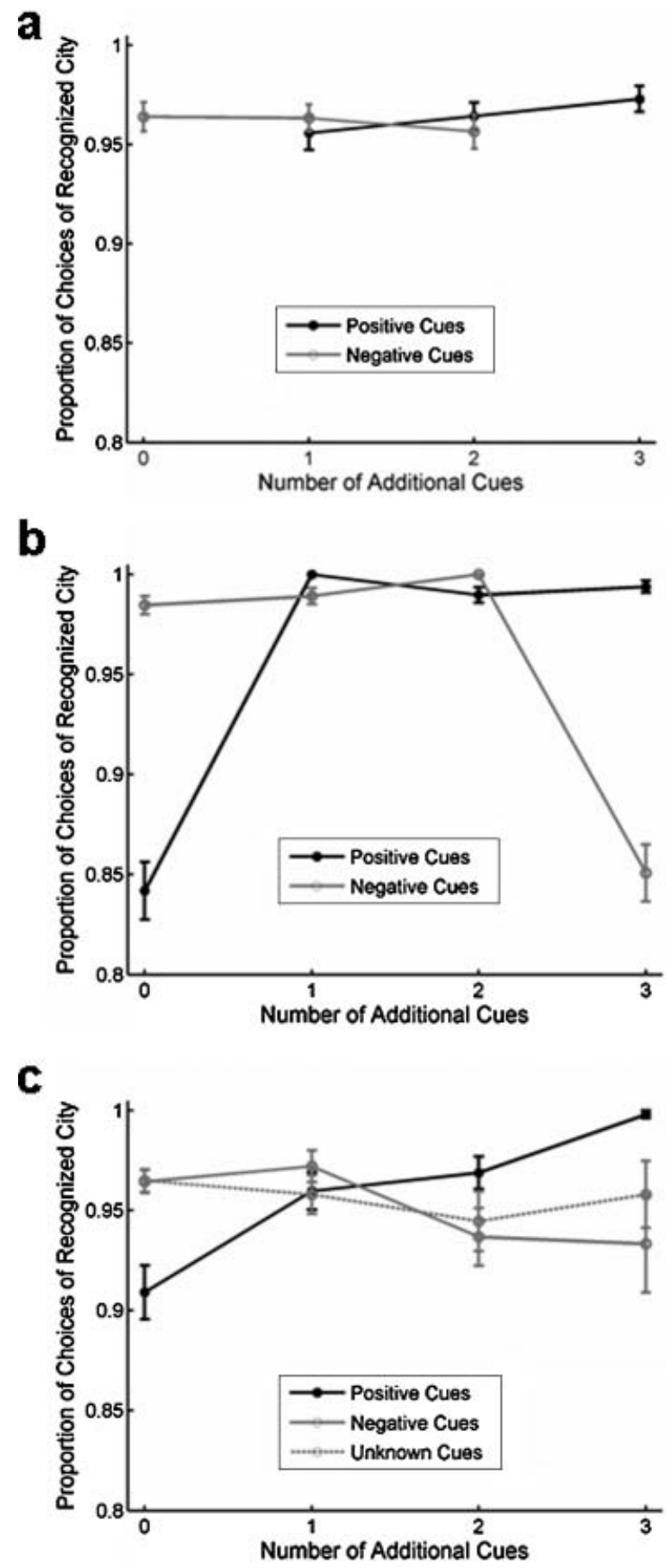

Figure 3(a-c). Proportion of choices of the recognized city as a function of the number of additional cues in Experiment 1 (a), Experiment 2 (b) and Experiment 3 (c). Error bars show \pm 1 standard error 
recognition. (But note that this result was in no way obvious before the experiment, as recognition was still contradicted by the most valid cue.) We addressed this possibility in Experiment 2.

\section{EXPERIMENT 2: REPLICATION WITH INCREASED AMOUNT OF EVIDENCE CONTRADICTING RECOGNITION}

Experiment 2 constituted an even tougher challenge for the recognition heuristic and tested it in the situation in which recognition was contradicted by three cues that participants in the pre-studies had mentioned as reliable indicators of city size. To carry out such a test, we changed the cue profiles that participants learned about recognized cities in Experiment 1 such that they now involved two cities that had negative values on all three cues. In all other respects, Experiment 2 was identical to Experiment 1.

\section{Method}

Participants and procedure

Forty students ( 25 females; mean age $=25.2$ years) took part in this experiment, which was conducted at the Max Planck Institute for Human Development in Berlin. Payment, design and procedure were identical to those in Experiment 1.

\section{Material}

The only difference between Experiments 1 and 2 was that for the cities of Brighton and York the positive values on the industry cue were replaced by negative ones (see Table 2).

\section{Results and discussion}

Cue knowledge, recognition and cue validity estimates

The mean proportion of critical pairs where one city was recognized was $.84(S D=0.19)$. On average, participants took 9.8 minutes $(S D=4.6)$ to learn the cue values perfectly and achieved, on average, an accuracy of $96 \%$ ( $S D=9$; range 67-100\%) in the subsequent memory task. Thirty of the 40 participants recalled the cue values perfectly. The response 'don't know' was chosen in $0.8 \%$ of the responses.

\section{Effect of additional cue knowledge on choice of recognized city}

Overall, participants' judgments followed recognition in, on average, 94\% $(S D=0.12)$ of the critical RU pairs where one city was recognized but the other was not. As shown in Figure 3b, the introduction of a cue pattern with exclusively negative cues had a large effect (though the same cities were used as in Experiment 1. Note that due to the modified cue patterns, there were now 4 categories for the amount of cue knowledge: 0,1 , 2, or 3 cues). Replicating the result of previous studies, but contrasting with Experiment 1, the proportion of choices of the recognized city was now strongly affected by the amount of additional cue knowledge, both for positive $\left[\chi^{2}(3, N=2020)=188.1, p=.001\right.$; two-tailed; $\left.w=0.31\right]$ and negative $\left[\chi^{2}(3, N=2020)=153.9\right.$, $p=.001$; two-tailed; $w=0.28]$ cue knowledge. A trend test (again using the critical threshold of 1.65 for $\alpha=.05)$ indicated a significant monotonic trend for both positive $(T=12.82)$ and negative $(T=11.41)$ cue knowledge.

To illustrate the effect of additional cue knowledge, take the cities of Brighton and York, for which the cue patterns learned in Experiments 1 and 2 differed. Whereas in Experiment 1, where participants had learned one positive cue value and two negative cue values in addition to recognition, $95.5 \%$ of the choices involving 
the two cities followed recognition, this percentage dropped to $85.9 \%$ in Experiment 2, where all three cues that participants learned about the cities contradicted recognition. Yet, the high proportion of choices of the recognized city indicates that the choices were still heavily influenced by recognition, given that all three additional cues contradicted recognition. Note that this figure is considerably higher than the $66 \%$ of choices following recognition reported for Richter and Späth's (2006) Experiment 2 in the same situation (but different domain) of recognition being contradicted by three additionally learned cues. Nevertheless, one might object that our results are still marred by ceiling effects. We would like to emphasize, however, that the high proportions of choices of the recognized city — close to 100\% — are in themselves an important result, if one recalls that in two of the conditions recognition is contradicted by one and two cues, respectively, that were judged by the participants as more valid than recognition. Even in this critical situation, recognition seemed to dominate the judgment.

As in Experiment 1 there were substantial individual differences. Forty-six per cent (18 of 39) of the participants with at least one case where a recognized city had no additional positive cue (but mostly negative cues, contradicting recognition; $M d n=18$ cases) always chose the recognized city. In other words, although on the aggregate level it looks as if participants' behaviour is at odds with the recognition heuristic, an individual analysis reveals that almost half of our participants made inferences in a way consistent with the heuristic.

\section{Discussion of Experiments 1 and 2}

Whereas we did not find that additional cue knowledge had an impact on the proportion of choices of a recognized city in Experiment 1, when we repeated the experiment with an increased amount of cue knowledge contradicting recognition in Experiment 2, we obtained a significant effect. In light of the high subjective validities of the three cues used, and viewed in comparison to the huge effects found in earlier studies (e.g. Bröder \& Eichler, 2006; Newell \& Shanks, 2004), the effect we observed was rather small. For illustration, when (induced) recognition was contradicted by three additional cues in Bröder and Eichler (2006), the recognized city was chosen, on average, merely $40 \%$ of the time - additional cue knowledge thus clearly dominated recognition. In our Experiment 2, by contrast, when (natural) recognition was contradicted by three cues, the recognized city was chosen, on average, $85 \%$ of the time. One possible explanation for this difference is that participants indeed put more weight on natural recognition than on experimentally induced recognition. On the other hand, the existing effect of additional cue knowledge in Experiment 2 shows that at least some of the participants incorporated this additional knowledge when forming their judgments. However, one could still object that the procedure of cue learning might have produced a demand effect that artificially promoted the use of the additional knowledge. The result of our Experiment 2, as well as those of Bröder and Eichler (2006), Newell and Shanks (2004), Newell and Fernandez (2006) and Richter and Späth (2006, Experiments 2 and 3), might thus be due to a demand effect rather than to a natural tendency to consider additional cue knowledge. To test this possible explanation, in Experiment 3 we did not have participants learn cue values. Instead, we related their choices to the already existing cue knowledge they expressed in a task administered after the inference task.

\section{EXPERIMENT 3: DOES NATURAL CUE KNOWLEDGE OVERRIDE NATURAL RECOGNITION?}

The goal of Experiment 3 was to minimize the experimenters' interference with participants' prior knowledge. Rather than testing the effect of experimentally induced cue knowledge, Experiment 3 involved naturally acquired recognition and already existing cue knowledge, thus minimizing the risk of creating a demand effect. To capture people's natural cue knowledge, we again relied on the three cues that were identified in the pre-studies as subjectively highly valid, and we assessed people's knowledge on these cues. 


\section{Method}

\section{Participants}

Forty students ( 25 females; mean age $=25.6$ years) took part in this experiment, which was conducted at the Max Planck Institute for Human Development in Berlin. The payment was identical to that in Experiments 1 and 2.

\section{Materials}

In this experiment, the cities of Manchester and Liverpool were replaced by Aberdeen and Bristol because the results of Pre-study 2 suggested that unambiguous cue knowledge (rather than 'don't know' responses) would be more likely for these two cities. The other $\mathrm{R}$ cities as well as the $\mathrm{U}$ and the filler cities were identical to those used in Experiments 1 and 2.

\section{Procedure}

The procedure and instructions were identical to those in Experiments 1 and 2, with the exception that there was no cue knowledge learning. Hence, the experiment began with the inference task, which was followed by the recognition task. In the knowledge task following the recognition task, participants indicated their knowledge about the six $\mathrm{R}$ cities on the three cues (industry, international airport and soccer team) by responding with 'yes', 'no', or 'don't know'. As in the previous experiments, participants' responses in this task were used as an independent variable to classify their choices in terms of the amount of additional knowledge about the recognized city in an RU pair. Participants were encouraged to be as accurate as possible in their responses and received an additional $10 \varnothing$ (13ф U.S.) for every correct response, and lost the same amount for every incorrect response ('don't know' responses, however, were not penalized).

\section{Results}

Cue knowledge, recognition and cue validity estimates

The mean proportion of critical pairs where one city but not the other was recognized was $.78(S D=0.23)$, which did not differ significantly from Experiments 1 and $2[F(2,117)=1.16, p=.32]$. In retrospect, we can thus say that the learning task in Experiments 1 and 2 does not seem to have affected participants' responses in the recognition task. The cue values that the participants indicated most frequently for the six cities in the knowledge task ('yes', 'no', or 'don't know') are reported in Table 2. Note that with the exception of the industry cue of Nottingham, these modal cue values agreed with those obtained in Pre-study 2. Overall, the option 'don't know' was chosen in $25 \%$ of the responses. This proportion was higher than in Experiments 1 and 2, probably due to the facts that in Experiment 3 the cue knowledge was not explicitly learned prior to the experiment and that participants had no incentive to avoid a 'don't know' answer when they were unsure about the accuracy of their cue knowledge. The estimated cue validities were not different from those in Experiments 1 and $2[F(4,115)=0.63, p=.64]$, indicating that teaching additional cue knowledge did not affect the perceived validity of the cues.

\section{Effect of additional cue knowledge on choice of recognized city}

Overall, participants' judgments followed recognition in, on average, 95.5\% $(S D=0.08)$ of the critical RU pairs where one city was recognized but the other was not. Figure $3 \mathrm{c}$ shows the proportion of choices of the recognized city as a function of the amount of positive, negative and unknown cue values, respectively, for the recognized city. Note that due to the presence of unknown cue values, the lines for positive and negative cue values are not symmetrical. As in Experiment 2, the proportion of choices of the recognized city varied as 
a function of the amount of both positive $\left[\chi^{2}(3, N=1862)=51.1, p=.001\right.$, two-tailed; $\left.w=0.17\right]$ and negative cue knowledge $\left[\chi^{2}(3, N=1,862)=8.2, p=.04\right.$, two-tailed; $\left.w=0.07\right]$ that was available in addition to recognition. However, the effect size of positive cue knowledge was rather small [according to Cohen's (1988) conventions, $w=0.17$ is a small to medium effect size], and only about half as large as in Experiment 2 . The number of unknown cue values had no effect on the proportion of choices of the recognized city $\left[\chi^{2}(3\right.$, $N=1862)=2.2, p=.54$, two-tailed; $w=0.03]$. Also as in Experiment 2, there was a monotonic trend for positive $(T=6.89)$, as well as negative $(T=2.15)$ cue knowledge, but not for the number of unknown cue values $(T=1.14$; again using a critical threshold of 1.65 for $\alpha=.05)$.

As in Experiments 1 and 2, we performed an individual analysis that included those participants with at least one case where a recognized city had no additional positive, but at least one cue (and usually more) contradicting recognition $(M d n=10$ cases). Of these participants, 48\% (15 of 33) always chose the recognized city, defying the effect of additional cue knowledge apparent on the aggregate level. Again, this result is consistent with the hypothesis that these individuals used recognition in a noncompensatory way and ignored further cue knowledge - as predicted by the recognition heuristic.

To summarize, in contrast to previous studies, and to our own Experiments 1 and 2, in Experiment 3 we examined the influence of additional cue knowledge on the use of recognition, without inducing this knowledge. Although we thus ruled out the possibility that the effects of additional cue knowledge were caused by a demand effect (as the knowledge about cues was assessed only after the inference task), the additional cue knowledge clearly affected the extent to which a recognized city was chosen over an unrecognized one. Compared to Experiment 2, however, the effect was smaller. The issue of a demand effect will be further discussed in the next section. Moreover, although on the aggregate level additional cue knowledge shows an effect that is inconsistent with the recognition heuristic, when looking at individual participants, we find a large proportion of participants where the additional knowledge they had available had no impact on their binary choices.

\section{ADDITIONAL ANALYSES}

Additional analyses across the three experiments were conducted to examine further (a) the difference between induced and natural cue knowledge and (b) individual differences in how recognition is used in the face of contradictory further cue knowledge.

\section{Are natural and induced cue knowledge used differently?}

To examine the differential effects of induced and natural cue knowledge on the use of recognition, we directly compared the effects observed in Experiments 2 and 3, where significant effects were obtained. Here we analysed the proportion of choices of the recognized city as a function of the net sum of the cue values known for a recognized city (i.e. including both positive and negative cues), rather than as a function of the number of positive or negative cues (as we had done in Figures $3 a-c){ }^{7}$ By looking at the net sum of the evidence provided by the cues we made the assumption that a positive value on one cue can be compensated

\footnotetext{
${ }^{7}$ This was necessary because Experiments 2 and 3 differed considerably in terms of the number of observations that were available for cities with zero, one, two or three positive cues. In addition, whereas in Experiment 2 the numbers of positive and negative cues were practically complementary, this was not the case in Experiment 3. To illustrate, when a city in Experiment 2 had only one positive cue, the remaining two cues were almost always two negative ones. In Experiment 3, by contrast, due to the higher number of unknown cue values, when a city had only one positive cue, it had either zero, one, or two negative cues (depending on the number of unknown cue values). Therefore, to make the results of Experiments 2 and 3 more comparable, we analysed participants' inferences using the cue sum as the independent variable. Note, moreover that we made the reasonable assumption that no knowledge was available for the unrecognized cities.
} 


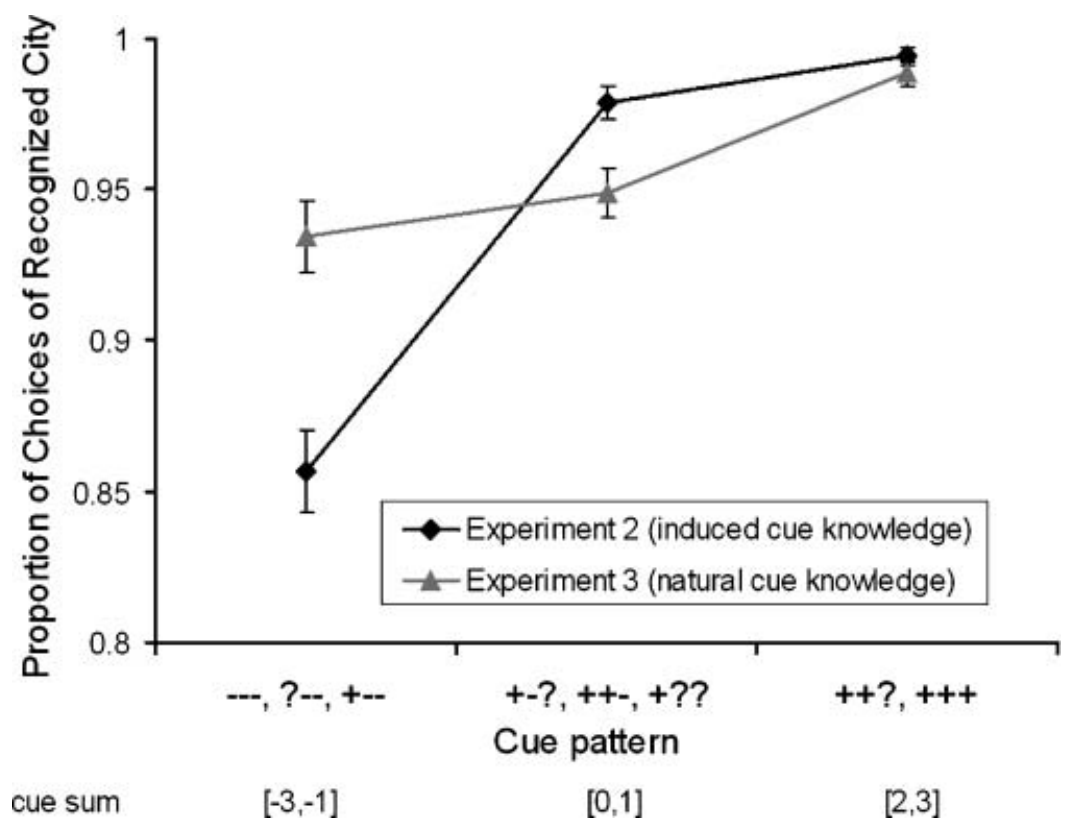

Figure 4. Proportion of choices of the recognized city as a function of the sum of positive and negative cue values, separately for Experiments 2 and 3. The numbers of choices were $n=656,664$ and 700 for Experiment 2, and $n=442$, 722 and 698 for Experiment 3. '+' denotes a positive cue value (i.e. 'yes'), '-' denotes a negative cue value (i.e. 'no'), '?' denotes an unknown cue value (i.e. 'don't know'). Error bars show \pm 1 standard error

for by a negative, but not by an unknown value on a another cue. To illustrate, the cue pattern ' ++- ' (i.e. 'yes,' 'yes,' and 'no' on the first, second, and third cue, respectively) has a cue sum of $1+1-1=1$, and the cue pattern '++?' (i.e. 'yes,' 'yes,' and 'don't know' on the first, second, and third cue, respectively) has a cue sum of $1+1+0=2$, although both cue patterns contain two positive cue values. Figure 4 shows the proportion of choices of the recognized city across three levels of cue sums, binned such that for both experiments, comparable numbers of choices were available for each level.

As can be seen, compared to when people have to rely on knowledge they acquired outside the laboratory (natural knowledge; Experiment 3), teaching cue information during the experiment (induced knowledge; Experiment 2) seems to generally amplify the effect of cue knowledge-consistent with the hypothesis that teaching cue knowledge creates a demand effect. The proportion of choices of the recognized city was lower when the cue knowledge was mainly negative (cue patterns,$--- ?--,+--$ ), and higher when it was mainly positive $(++?,+++)$. To quantify these differences, we contrasted the proportion in the lowest level with the proportion in the highest level, separately for Experiments 2 and 3. For Experiment 2, we obtained an effect size $h$ (Cohen, 1988) of 0.63, a medium to large effect size for additional cue knowledge, whereas for Experiment $3 h$ equalled 0.30, a small to medium effect size.

This analysis suggests that inducing cue knowledge indeed increases the weight given to this knowledge relative to recognition: When the cue knowledge was negative (i.e. contradicting recognition), the proportion of choices of the recognized city was lower with induced than with natural cue knowledge, whereas when the cue knowledge was positive (i.e. supporting recognition), the proportion of choices of the recognized city was higher with induced than with natural cue knowledge. Interestingly, the effect of induced cue knowledge was observed even though the proportion of 'orthodox' users of the recognition heuristic (i.e. those that chose the recognized city irrespective of the presence of other cue knowledge) was similar in Experiments $2(46 \%)$ and $3(48 \%)$. The effect is thus probably due to differences in the compensatory users of recognition, who seem to 

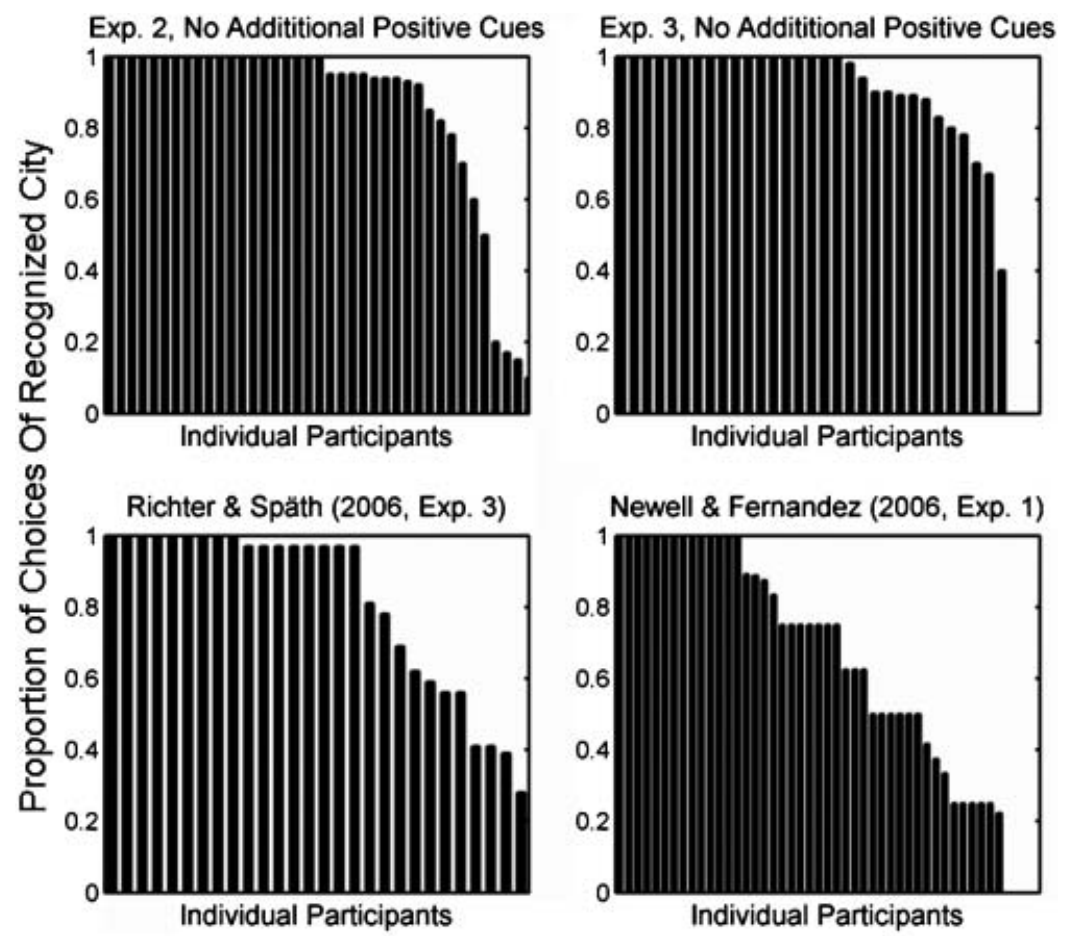

Individual Participants

Figure 5. Distribution of individual proportions of choices of the recognized city when no additional positive cue knowledge was available in Experiments 2 and 3, shown in the upper row, and in a reanalysis of Richter and Späth (2006,

Exp. 2; 28 participants) and Newell and Fernandez (2006, Exp. 1; 48 participants), shown in the lower row

have paid more attention to additional cues when the cue knowledge was induced (compared to when it was natural).

Yet, we note that there is an alternative to the demand effect explanation proposed above: In Experiment 3, participants may have used any knowledge they had, not only and necessarily the cues that we asked them about afterward. Hence, the three cues we used to assess people's cue knowledge are only indicators of additional knowledge, and effects may be attenuated by the 'noise' introduced by other knowledge. In contrast, learning cues beforehand may direct attention to them as potentially useful in the subsequent judgment task. If participants choose to rely on them, effects appear stronger. We emphasize, however, that at no point during the experiment was the potential relevance of the cues for the inference task mentioned. Regardless of whether the explanation in terms of a demand effect or in terms of noise holds, we conclude that in finding that additional cue knowledge has an effect when demand effects are ruled out our study supports the observation of earlier studies that additional cue knowledge affects inferences. Importantly, however, the effect seems to vary considerably among participants.

\section{Individual differences in the use of recognition}

Additional cue knowledge had an effect in Experiments 2 and 3; yet in all experiments, we observed that a substantial number of participants always chose the recognized city and thus did not seem to be affected by additional cue knowledge. For illustration, the upper row of Figure 5 shows for individual participants in Experiments 2 and 3 the proportions of choices of the recognized city when no additional positive cue 
knowledge was known (and where most additional cues suggested that the recognized city is small). As can be seen, in both experiments, half of the participants always chose the recognized city. At the same time, there was a (small) group of participants who seemed to decide systematically against the recognized city: In Experiment 2, there were four participants who chose the recognized city only 17\%, 10\%, 20\% and 29\% of the time, and in Experiment 3, two participants never chose the recognized city. One way to interpret the consistency of the participants who always chose the recognized city is that these participants used the recognition heuristic. That is, although the participants reported after the inference task to have additional relevant cue knowledge about a recognized city that contradicted recognition, they appeared to have stopped information search after assessing recognition for the two objects; other participants, by contrast, integrated that other knowledge into their inferences.

Importantly, the existence of large and sometimes dramatic individual differences in the use of recognition does not seem restricted to our experiments: the lower row of Figure 5 shows the data of two other experiments with natural recognition and inferences from memory that found additional cue knowledge to have an effect on the aggregate level (Newell \& Fernandez, 2006; Richter \& Späth, 2006). Depicted are the individual participants' proportions of choices of the recognized object when recognition was contradicted by three (out of three) and one (out of one) additional cues, respectively. As can be seen, there are large individual differences as well, and a substantial number of participants who chose the recognized object irrespective of conflicting cue knowledge.

The impact of perceived recognition validity

What could be the reason for the large individual differences in the use of recognition? One factor that could impact a person's processing of recognition is her perceived validity of recognition for the given inference task; two possibilities should be distinguished: (a) that recognition's relative validity (compared to other cues), or (b) that recognition's absolute validity is key. First, recognition might be used differently by participants who perceived recognition to be the most valid of the available cues than by participants who perceived a different cue as most valid. When comparing the inferences of these two groups in our Experiments 1-3, however, there was no such effect. As can be seen in Figure 6, the proportion of choices of the recognized city varied across different levels of additional cue knowledge irrespective of whether recognition was estimated to be the most valid cue (of the four) or not.

Alternatively, it is possible that it is the absolute value of recognition's validity that determines how recognition is used for an inference. Therefore, in a further analysis, we compared participants (collapsing across the three experiments) who always chose the recognized city (54 of 120 participants,
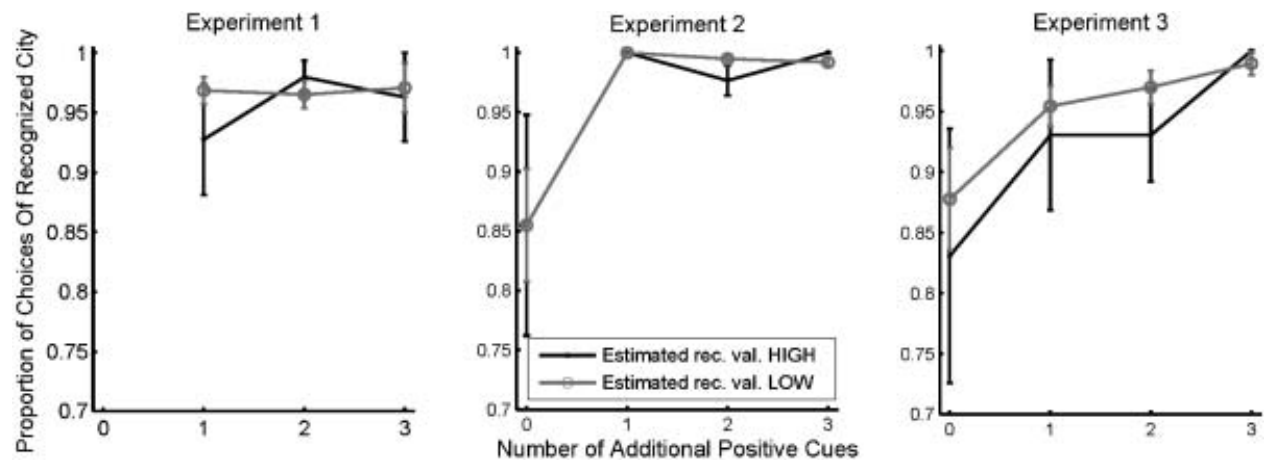

Figure 6. Proportion of choices of the recognized city across the different levels of additional positive cue knowledge, separately for participants who estimated recognition to be the most valid cue (Estimated rec. val. HIGH) and all other participants (Estimated rec. val. LOW). Error bars show \pm 1 standard error 
noncompensatory group) with all others (51, compensatory group). Only those participants were included in this analysis for whom there was at least one case for the two extreme levels of knowledge (i.e. 1 and 3 positive cues for Experiment 1, and 0 and 3 positive cues for Experiments 2 and 3); 15 participants remained unclassified. Interestingly, the noncompensatory users of recognition estimated it to be more valid (in absolute terms) than the compensatory users $[M=73.7, S D=13.1$ vs. $M=68.0, S D=14.5 ; t(103)=2.09$, $p=.04 ; d=.41]$. In other words, the absolute value of recognition's perceived validity could be one factor fostering its noncompensatory processing. Similarly, collapsing participants in all three experiments (irrespective of their classification as compensatory or noncompensatory users) there was a small and positive correlation between estimated recognition validity and proportion of choices following recognition $(r=.12$, $p=.09$, one-tailed).

\section{GENERAL DISCUSSION}

We found evidence suggesting that recognition operates as a highly dominant cue in probabilistic inferences: Even when the recognition cue is not perceived as most valid and in addition is contradicted by a substantial amount of additional and relevant cue knowledge, there seems to be strong reliance on recognition. As the difference between Experiments 1 and 2 suggests, a comparatively large amount of negative evidence is necessary to override its impact. Importantly, this does not seem to be the case with induced recognition, which was used in earlier experiments (e.g. Bröder \& Eichler, 2006; Newell \& Shanks, 2004). An interesting result is that the dominating effect of recognition does not seem to depend on recognition being perceived as the most valid cue; in fact, participants estimated the cue validity of recognition to be lower than the validity of two of the three cues that they were taught in addition to recognition (although, at over .70, the absolute value of recognition's validity was still rather high). Nevertheless, we obtained some evidence that the absolute level of recognition's perceived validity might influence whether recognition is used in a compensatory or noncompensatory fashion.

Although cross-experiment comparisons have to be treated with caution, a direct comparison of our results with those, for instance, of Bröder and Eichler (2006) suggests that additional knowledge has stronger effects in tests involving both induced recognition and additional cue knowledge taught in the experiment than in tests involving naturally evolved recognition and knowledge that people have acquired outside the laboratory. Hence, previous experiments that used induced recognition and/or induced cue knowledge may have overstated the impact of additional cue information on probabilistic inferences. The same holds true for studies testing the recognition heuristic in domains with a low recognition validity.

To be sure, even in our Experiment 3, in which no cue knowledge was induced and thus all precautions were taken to rule out demand effects, we found additional cue knowledge to have a small, but reliable effect. This effect, however, was driven mainly by the fact that about one-half of the participants seem to have included additional cue information in their judgments; the other half chose the recognized city irrespective of additional cue knowledge, suggesting that they ignored the additional cues-as predicted by the recognition heuristic.

The need to make explicit such individual differences in decision behaviour has been emphasized repeatedly (e.g. Brehmer, 1994; Bröder, 2000b; Einhorn, 1970). And individual differences in strategy use have also been observed in experimental research on other noncompensatory inference heuristics, such as 'TTB' (Gigerenzer \& Goldstein, 1996). For example, Bröder (2000b, Experiment 1) showed that not all people use TTB in probabilistic inferences. But hypothesis rejections at the group level may throw out the baby with the bath water if individual strategy differences are not taken into account. Almost all experimental studies on TTB have shown that varying proportions of participants do adhere to this heuristic, depending on task demands and classification criteria (Bergert \& Nosofsky, 2007; Bröder, 2000b, 2003; Lee \& Cummins, 2004; Newell \& Shanks, 2003; Newell, Rakow, Weston, \& Shanks, 2004; Newell, Weston, \& Shanks, 2003; 
Rieskamp \& Hoffrage, 1999; Rieskamp \& Otto, 2006). Conversely, whereas the proportion of TTB users varies - often in an adaptive fashion-there are always at least some participants who seem to prefer other-compensatory or noncompensatory-strategies.

In light of these findings, the focus of research on TTB has shifted away from initial attempts to refute the notion that the heuristic is always used towards systematic explorations of factors that affect noncompensatory strategy use (such as information costs and cognitive capacity; Bröder, 2003; Bröder \& Schiffer, 2003, 2006). Richter and Späth (2006) proposed a similar shift for research on the recognition heuristic, summarizing: 'The conclusion that the recognition heuristic is not universally applied leaves us with the question of whether there are situations in which people use recognition in a noncompensatory way and, if so, which factors yield a noncompensatory use of recognition' (p. 160).

In research on TTB, a more refined approach that examines strategy use at the individual level has proven to be fruitful, as the boundary conditions of the theory continue to be refined. Likewise with the recognition heuristic, recent work has begun to systematically flesh out the boundary conditions of its use (e.g. Newell \& Shanks, 2004; Pachur \& Hertwig, 2006). In our view, it is now clear that the recognition heuristic-in particular in terms of the hypothesized noncompensatory use of recognition - is not used by all people all the time and under all circumstances, so we think it is time to reformulate the research question accordingly. For instance, one important task would be to identify factors driving individual differences in the use of the recognition heuristic. In the context of TTB, such an approach has led to promising results in the quest to better understand adaptive decision-making (Bröder, 2003; Mata, Schooler, \& Rieskamp, in press), and some encouraging insights are emerging for the recognition heuristic as well (Pachur, Mata, \& Schooler, 2006).

Individual differences pose a problem for theorizing and empirical studies. Theorizing has been largely 'nomothetic' in the decision-making domain, in the sense that the focus was on identifying general laws of information processing. On the other hand, some researchers have tried to identify personal preferences for processing styles in an 'idiographic' fashion, that is, they tried to explain strategy selection based on individual traits (e.g. Schunk \& Betsch, 2006; Zakay, 1990). If, however, personality differences consistently moderate the general laws, one is probably forced to model situation $\times$ person interaction in full-fledged theories of decision-making (Mischel \& Shoda, 1995). This is also a challenge for empirical research because viable methods for assessing strategy use have to be developed. We feel that such a research agenda is overdue since strategy differences between people appear to be the rule rather than marginal exceptions.

\section{Is noncompensatory processing of recognition due to constrained information retrieval?}

Our results clearly demonstrate a dominating effect of recognition over other probabilistic cues. But there are (at least) two ways such a dominating effect could come about, of which only one is, strictly speaking, consistent with Goldstein and Gigerenzer's (2002) description of the recognition heuristic. First, additional cue knowledge could fail to compensate for recognition information because no other cues are retrieved. This would be the mechanism envisaged by Goldstein and Gigerenzer and not a completely implausible one, given that recognition seems to have a retrieval primacy over other probabilistic cues (Pachur \& Hertwig, 2006). Alternatively, recognition could solely determine the judgment because, although all cues are retrieved, recognition is very heavily weighted. A possible reason for such a dominating weighting of recognition could be that people can assess an object's value on the recognition cue with higher confidence than its value on other cues. ${ }^{8}$ Although based on our current results we cannot ultimately decide between these two possibilities, our participants' explicit cue validity estimates do not suggest that recognition was weighted

\footnotetext{
${ }^{8}$ But note that participants had the option to indicate that they had low confidence in their cue knowledge by indicating 'don't know' in the memory task (Exp. 1-2) and the knowledge task (Exp. 3) - which they did only relatively rarely.
} 
particularly heavily (as two other cues were given higher validity estimates than recognition). It is of course possible that subjective cue weights are a function of something else than their perceived validities, for example the perceived applicability of a cue. As mentioned earlier, in pairs of objects where only one object is recognized, recognition is the only cue for which both values are known, whereas for the other cues values are known for the recognized object only. This may lead to a discounting of the weight given to further cue knowledge (compared to the recognition cue). Outcome data alone cannot distinguish between both accounts, and future studies may favour one of the explanations based on process data such as response times (Bergert \& Nosofsky, 2007; Bröder \& Gaissmaier, in press; Pachur \& Hertwig, 2006).

\section{CONCLUSION}

'The recognition heuristic follows particularly simple rules. Search extends only to recognition information, not to recall. Search is stopped whenever one object is recognized and the other is not; no further information is looked up about the recognized object. The simple decision rule is to choose the recognized object' (Goldstein \& Gigerenzer, 2002, p. 88). In our experiments, when recognition evolved outside the experimental setting and was a valid cue, about half of the participants seem to have applied a noncompensatory strategy that ignores further cues. In addition, our results suggest that the dominance of recognition is sensitive not only to whether recognition knowledge has been induced during the experiment, but also to whether the cue knowledge has been acquired within the experimental setting. Since individuals appear to differ greatly in their reliance on recognition for inferences (which is moderated but not completely determined by its perceived validity), the challenging future task is to identify the further determinants of strategy selection. Hence, as in other areas of decision research, here too it appears worthwhile to 'combine an idiographic and nomothetic approach' (Einhorn, 1970, p. 228).

\section{APPENDIX}

Table A1 Recognition rates of the cities used in Experiments 1-3

\begin{tabular}{llccc}
\hline Item type & City & Experiment 1 & Experiment 2 & Experiment 3 \\
\hline R high & Aberdeen & 0.95 & 0.98 & - \\
& Bristol & 0.90 & 0.93 & - \\
& Manchester & - & - & 1 \\
R intermediate & Liverpool & - & 1 & 0.98 \\
& Nottingham & 1 & 0.93 & 0.85 \\
R low & Sheffield & 0.85 & 0.90 & 0.93 \\
& Brighton & 0.93 & 1 & 0.93 \\
$\mathrm{U}$ & York & 0.88 & 0.18 & 0.25 \\
& Southend-on-Sea & 0.13 & 0.03 & 0.15 \\
& Stockport & 0.08 & 0.20 & 0.30 \\
& Stoke-on-Trent & 0.15 & 0.18 & 0.25 \\
& Swindon & 0.13 & 0.05 & 0.10 \\
& Walsall & 0.03 & 0.15 & 0.23 \\
& West Bromwich & 0.23 & 0.13 & 0.18 \\
& Poole & 0.13 & 0.03 & 0.10 \\
& Oldbury/Smethwick & 0.05 & 0.10 & 0.13 \\
& Huddersfield & 0.08 & 0.18 & 0.18 \\
\hline
\end{tabular}

Note: R, recognized; $\mathrm{U}$, unrecognized (as classified based on the results in the pre-studies); high, intermediate and low refer to the number of additional positive cues ( 3,2 and 1 positive cues, respectively). 


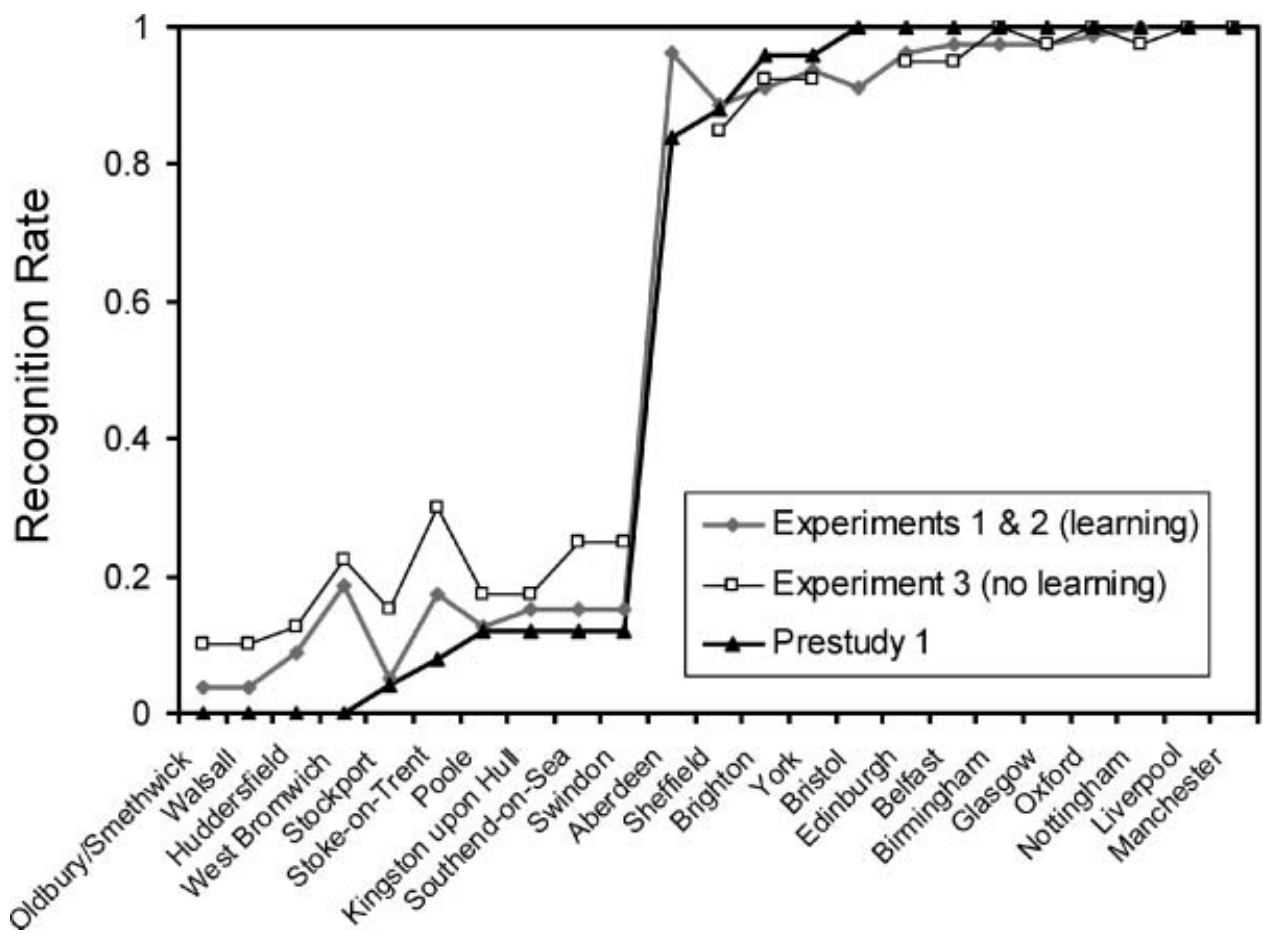

Figure A1. Recognition rates for the cities used in the experiments

Were participants' recognition rates affected by experimental familiarity to the city names?

Recall that we had participants indicate whether or not they have heard of a city name before participating in the experiment after the experiment. By using these recognition assessments as a basis for testing the recognition heuristic, we assumed that participants were able to disregard the familiarity with the city names produced by the exposures during the experiment. How reasonable is this assumption? Evidence that people are indeed capable of screening out experimental familiarity comes from at least two sources: First, none of the studies that controlled for the order of recognition and judgment tasks found a significant effect of task order (Pohl, 2006; Pachur \& Hertwig, 2006). Second, Figure A1 compares (for the cities used in Experiments 1-3) the recognition rates obtained in Pre-study 1 - where participants saw the city names only once-with the recognition rates obtained in Experiments 1-3-where each unrecognized city was presented in total around 12 times before a recognition judgment was made. Although the recognition rates for the very unfamiliar cities obtained in Experiments 1-3 are slightly higher than in the pre-study (interestingly, in particular in Experiment 3), the difference is rather small. Moreover, note that in spite of having been presented a dozen times, the highly recognized cities seemed to be slightly less recognized in Experiments 1-3 than in Pre-study 1, suggesting that the differences are also due to a normal regression effect.

\section{ACKNOWLEDGEMENTS}

Our thanks go to Bryan Bergert Wolfgang Gaissmaier, Gerd Gigerenzer, Daniel Goldstein, and Lael Schooler for many constructive comments. In addition, we are grateful to Tobias Richter and Ben Newell for making available the raw data of their experiments. 


\section{REFERENCES}

Bergert, F. B., \& Nosofsky, R. M. (2007). A response-time approach to comparing generalized rational and take-the-best models of decision making. Journal of Experimental Psychology: Learning, Memory, and Cognition, 31, $107-129$.

Brehmer, B. (1994). The psychology of linear judgment models. Acta Psychologica, 87, 137-154.

Bröder, A. (2000a). A methodological comment on behavioral decision research. Psychologische Beiträge, 42, 645-662.

Bröder, A. (2000b). Assessing the empirical validity of the "take-the-best" heuristic as a model of human probabilistic inference. Journal of Experimental Psychology: Learning, Memory, and Cognition, 26, 1332-1346.

Bröder, A. (2003). Decision making with the "adaptive toolbox": Influence of environmental structure, intelligence, and working memory load. Journal of Experimental Psychology: Learning Memory, and Cognition, 29, 611-625.

Bröder, A., \& Eichler, A. (2006). The use of recognition information and additional cues in inferences from memory. Acta Psychologica, 121, 275-284.

Bröder, A., \& Gaissmaier, W. (in press) Sequential processing of cues in memory-based multi-attribute decisions. Psychonomic Bulletin and Review.

Bröder, A., \& Schiffer, S. (2003). Take the best versus simultaneous feature matching: Probabilistic inferences from memory and effects of representation format. Journal of Experimental Psychology: General, 132, $277-293$.

Bröder, A., \& Schiffer, S. (2006). Stimulus format and working memory in fast and frugal strategy selection. Journal of Behavioral Decision Making, 19, 361-380.

Cohen, J. (1988). Statistical power analysis for the behavioral sciences (2nd ed.). Hillsdale, NJ: Erlbaum.

Einhorn, H. J. (1970). The use of nonlinear, noncompensatory models in decision making. Psychological Bulletin, 73 , 221-230.

Ford, J. K., Schmitt, N., Schechtman, L. S., Hults, B. M., \& Doherty, M. L. (1989). Process tracing methods: Contributions, problems, and neglected research questions. Organizational Behavior and Human Decision Processes, 43, 75-117.

Gigerenzer, G., \& Goldstein, D. (1996). Reasoning the fast and frugal way: Models of bounded rationality. Psychological Review, 103, 650-669.

Gigerenzer, G., Hoffrage, U., \& Kleinbölting, H. (1991). Probabilistic mental models: A Brunswikian theory of confidence. Psychological Review, 98, 506-528.

Gigerenzer, G., Todd, P. M., \& the ABC Research Group. (1999). Simple heuristics that make us smart. New York: Oxford University Press.

Goldstein, D. G., \& Gigerenzer, G. (2002). Models of ecological rationality: The recognition heuristic. Psychological Review, 109, 75-90.

Grice, H. P. (1975). Logic and conversation. In P. Cole , \& J. Morgan (Eds.), Syntax and semantics (Vol. 3, pp. 41-58). New York: Academic Press.

Hertwig, R., \& Todd, P. M. (2003). More is not always better: The benefits of cognitive limits. In D. Hardman , \& L. Macchi (Eds.), Thinking: Psychological perspectives on reasoning, judgment and decision making (pp. 213-231). Chichester, England: Wiley.

Hilton, D. (1995). The social context of reasoning: Conversational inference and rational judgment. Psychological Bulletin, 118, 248-271.

Jacoby, L. L., Kelley, C. M., Brown, J., \& Jasechko, J. (1989). Becoming famous overnight: Limits on the ability to avoid unconscious influences of the past. Journal of Personality and Social Psychology, 56, 326-338.

Keeney, R., \& Raiffa, H. (1993). Decisions with multiple objectives: Preferences and value tradeoffs. Cambridge: Cambridge University Press.

Lee, M. D., \& Cummins, T. D. R. (2004). Evidence accumulation in decision making: Unifying the "take the best" and the "rational" models. Psychonomic Bulletin and Review, 11, 343-352.

Marewski, J. N., Gaissmaier, W., Dieckmann, A., Schooler, L. J., \& Gigerenzer, G. (2005). Ignorance-based reasoning? Applying the recognition heuristic to elections. Paper Presented at the 20th Biennial Conference on Subjective Probability, Utility and Decision Making, Stockholm.

Marewski, J. N., Gaissmaier, W., Schooler, L. J., Goldstein, D. G., \& Gigerenzer, G. (2007). When do people rely on recognition in inferential judgments? Unpublished manuscript.

Mata, R., Schooler, L., \& Rieskamp, J. (in press). The aging decision maker: Cognitive aging and the adaptive selection of decision strategies. Psychology \& Aging. DOI: 10.1037/0882-7974

Mischel, W., \& Shoda, Y. (1995). A cognitive-affective system theory of personality: Reconceptualizing situations, dispositions, dynamics, and invariance in personality structure. Psychological Review, 102, 246-268.

Newell, B. R., \& Fernandez, D. (2006). On the binary quality of recognition and the inconsequentiality of further knowledge: Two critical tests of the recognition heuristic. Journal of Behavioral Decision Making, 19, 333-346. 
Newell, B. R., Rakow, T., Weston, N. J., \& Shanks, D. R. (2004). Search strategies in decision making: The success of success. Journal of Behavioral Decision Making, 17, 117-137.

Newell, B. R., \& Shanks, D. R. (2003). Take the best or look at the rest? Factors influencing "one-reason" decision making. Journal of Experimental Psychology: Learning, Memory, and Cognition, 29, 53-65.

Newell, B. R., \& Shanks, D. R. (2004). On the role of recognition in decision making. Journal of Experimental Psychology: Learning, Memory, and Cognition, 30, 923-935.

Newell, B. R., Weston, N. J., \& Shanks, D. R. (2003). Empirical tests of a fast-and-frugal heuristic: Not everyone "takes-the-best." Organizational Behavior and Human Decision Processes, 91, 82-96.

Oppenheimer, D. M. (2003). Not so fast! (and not so frugal!): Rethinking the recognition heuristic. Cognition, 90, B1-B9.

Oppenheimer, D. M. (2004). Spontaneous discounting of availability in frequency judgment tasks. Psychological Science, $15,100-105$.

Pachur, T., \& Biele, G. (2007). Forecasting from ignorance: The use and usefulness of recognition in lay predictions of sports events. Acta Psychologica, 125, 99-116.

Pachur, T., \& Hertwig, R. (2006). On the psychology of the recognition heuristic: Retrieval primacy as a key determinant of its use. Journal of Experimental Psychology: Learning, Memory, and Cognition, 32, 983-1002.

Pachur, T., Mata, R., \& Schooler, L. (2006). Aging and the use of recognition in decision making. Poster Presented at the Annual Meeting of the Society for Judgment and Decision Making, Houston, Texas.

Pfanzagl, J. (1974). Allgemeine Methodenlehre der Statistik. Berlin: De Gruyter.

Pohl, R. (2006). Empirical tests or the recognition heuristic. Journal of Behavioral Decision Making, 19, $251-271$.

Richter, T., \& Späth, P. (2006). Recognition is used as one cue among others in judgment and decision making. Journal of Experimental Psychology: Learning, Memory, and Cognition, 32, 150-162.

Rieskamp, J., \& Hoffrage, U. (1999). When do people use simple heuristics and how can we tell? In G. Gigerenzer, P. M. Todd, \& The ABC Research Group (Eds.), Simple heuristics that make us smart (pp. 141-167). New York: Oxford University Press.

Rieskamp, J., \& Otto, P. (2006). SSL: A theory of how people learn to select strategies. Journal of Experimental Psychology: General, 135, 207-236.

Scheibehenne, B., \& Bröder, A. (2007). Predicting Wimbledon tennis results 2005 by mere player name recognition. International Journal of Forecasting, 23, 415-426.

Schooler, L. J., \& Hertwig, R. (2005). How forgetting aids heuristic inference. Psychological Review, 112, 610-628.

Schunk, D., \& Betsch, C. (2006). Explaining heterogeneity in utility functions by individual differences in decision modes. Journal of Economic Psychology, 27, 386-401.

Schwarz, N., Bless, H., Strack, F., Klumpp, G., Rittenauer-Schatka, H., \& Simons, A. (1991). Ease of retrieval as information: Another look at the availability heuristic. Journal of Personality and Social Psychology, 45, 513-523.

Serwe, S., \& Frings, C. (2006). Who will win Wimbledon 2003? The recognition heuristic in predicting sports events. Journal of Behavioral Decision Making, 19, 321-332.

Simon, H. A. (1955). A behavioral model of rational choice. Quarterly Journal of Economics, 69, 99-118.

Snook, B., \& Cullen, R. M. (2006). Recognizing national hockey league greatness with an ignorance-based heuristic. Canadian Journal of Experimental Psychology, 60, 33-43.

Tversky, A. (1972). Elimination by aspects: A theory of choice. Psychological Review, 79, 281-299.

Volz, K. G., Schooler, L. J., Schubotz, R. I., Raab, M., Gigerenzer, G., \& von Cramon, D. Y. (2006). Why you think Milan is larger than Modena: Neural correlates of the recognition heuristic. Journal of Cognitive Neuroscience, 18, 1924-1936.

Zakay, D. (1990). The role of personal tendencies in the selection of decision-making strategies. Psychological Record, 40, 207-213.

Authors' biographies:

Thorsten Pachur received his Ph.D. from the Free University in Berlin in 2006. He was predoctoral and a postdoctoral fellow at the Max Planck Institute for Human Development, Center for Adaptive Behavior and Cognition in Berlin and now works as a Research Scientist at the University of Basel. His research interests include memory processes in judgment and decision-making, frequency cognition and the psychology of risky choice.

Arndt Bröder received his Ph.D. from the University of Heidelberg in 1999. Following positions as a Lecturer in Bonn and as a visiting researcher at the Center for Adaptive Behavior and Cognition in Berlin, he is now a Professor for General Psychology in Bonn. His main research interests comprise methodology, memory and decision-making, and the interplay of the latter two. 
Julian Marewski received his diploma in Psychology in 2005 and is working as predoctoral research fellow at the Max Planck Institute for Human Development, Center for Adaptive Behavior and Cognition in Berlin. His main research interests include the use of recognition and retrieval fluency in decision-making.

Authors' addresses:

Thorsten Pachur, Cognitive and Decision Sciences, Faculty of Psychology, University of Basel, Basel, Switzerland

Arndt Bröder, Department of Psychology, University of Bonn, and Max Planck Institute for Research on Collective Goods, Bonn, Germany

Julian N. Marewski, Center for Adaptive Behavior and Cognition, Max Planck Institute for Human Development, Berlin, Germany 